Submitted to ApJ

Preprint typeset using LATEX style emulateapj v. 08/22/09

\title{
THE TIDAL EVOLUTION OF LOCAL GROUP DWARF SPHEROIDALS
}

\author{
Jorge Peñarrubia ${ }^{1}$, Julio F. Navarro ${ }^{2}$ \& Alan W. McConnachie \\ Department of Physics and Astronomy, University of Victoria, 3800 Finnerty Rd., Victoria, BC, V8P 5C2, Canada \\ Submitted to ApJ
}

\begin{abstract}
We use N-body simulations to study the evolution of dwarf spheroidal galaxies (dSphs) driven by galactic tides. We adopt a cosmologically-motivated model where dSphs are dark matter-dominated systems on eccentric orbits whose stellar component may be approximated by a King model embedded within an NFW halo. We find that these NFW-embedded King models are extraordinarily resilient to tides; the density profile of the stellar component still resembles a King model even after losing more than $99 \%$ of the stars. As tides strip the galaxy, the stellar luminosity, $L$, velocity dispersion, $\sigma_{0}$, central surface brightness, $\Sigma_{0}$, and core radius, $R_{c}$, decrease monotonically. Remarkably, we find that the evolution of these parameters is solely controlled by the total amount of mass lost from within the luminous radius. Of all parameters, the core radius is the least affected: after losing $99 \%$ of the stars, $R_{c}$ decreases by just a factor of $\sim 2$, implying that even in the event of extreme mass loss the core radius is a robust measure of the original size of the system. Contrary to naive expectations, tides tend to make dSphs more dark-matter dominated. This is because the tightly bound central dark matter "cusp" is more resilient to disruption than the comparatively more loosely bound "cored" King profile. We examine whether tidal effects may help to explain the extremely large mass-tolight ratios of some of the newly-discovered ultra-faint Milky Way dwarfs as tidal remnants of once brighter systems. Although dSph tidal evolutionary tracks parallel the observed scaling relations in the luminosity-radius plane, they predict too steep a change in velocity dispersion compared with the observational estimates hitherto reported in the literature. The ultra-faint dwarfs are thus unlikely to be the tidal remnants of systems like Fornax, Draco, or Sagittarius. Despite spanning four decades in luminosity, dSphs appear to inhabit halos of comparable peak circular velocity, lending support to scenarios that envision dwarf spheroidals as able to form only in halos above a certain mass threshold. Subject headings:
\end{abstract}

\section{INTRODUCTION}

Dwarf spheroidal galaxies (dSphs) are the faintest objects believed to be dominated by dark matter, and as such play a critical role in galaxy formation models. Because of their extremely low luminosity, this population of dwarf galaxies has been studied in detail almost exclusively in the Local Group (LG). The prototype dSph is a low surface brightness spheroid of old stars, with little detectable gas and few signs of ongoing or very recent star formation. They have luminosities and velocity dispersions comparable to globular clusters, but their much larger size denote the presence of large amounts of dark matter and set them apart from star clusters.

The best studied ("classical") Local Group dSphs span roughly two decades in luminosity, $3 \times 10^{5} \lesssim L / L_{\odot} \lesssim$ $3 \times 10^{7}$, from the brightest (Sagittarius) to the faintest (Draco). In contrast to the large range spanned in $L$, all of these systems have similar velocity dispersion $\sigma \sim 7-10 \mathrm{~km} / \mathrm{s}$, and share a characteristic value for the dynamically-inferred mass within the luminous radius, of order $\sim 10^{7} M_{\odot}$ (for a review, see, e.g., Mateo 1998).

This characteristic mass scale is interesting from a cosmological perspective (see, e.g., Gilmore et al 2007 for a recent discussion). Indeed, in the current $\Lambda \mathrm{CDM}$ paradigm of structure formation, dark matter halos of such mass outnumber the known dSphs by a factor of

${ }^{1}$ Email: jorpega@uvic.ca

${ }^{2}$ Fellow of the Canadian Institute for Advanced Research
10 to 100 , a result that highlights the low formation efficiency of visible galaxies in low mass halos (Kauffmann, White \& Guiderdoni 1993, Cole et al 1994, Klypin et al 1999, Moore et al 1999). A number of mechanisms have been proposed to explain the scarcity of sub-luminous galaxies in the $\Lambda \mathrm{CDM}$ scenario; these typically appeal to feedback effects associated with stellar evolution, as well as to heating from external UV radiation in order to hinder the cooling of gas and its transformation of stars in low-mass halos (Efstathiou 1992, Bullock et al 2000, Somerville 2002, Benson et al 2002).

Although these models can, with plausible assumptions, reconcile the faint end of the galaxy luminosity function with the low-mass end of the dark matter halo mass function, they have so far provided little insight into the origin of the structural properties of dSphs. For example, the large variation in mass-to-light ratio between dSphs noted above (Mateo 1998); the well-defined "King-like" core-halo structure of the stars (e.g. Irwin \& Hatzidimitriou 1995); the intriguing presence of multiple dynamical stellar components (Tolstoy et al. 2004; Battaglia et al. 2006; Ibata et al. 2006); the near constancy of their velocity dispersion profiles (e.g. Wilkinson et al. 2004; Muñoz et al. 2005; Walker et al. 2006, 2007; Koch et al. 2007a,b; Mateo et al. 2007) -all of these are well established observational facts of unclear origin and so far lacking definitive theoretical underpinning.

Clues to these questions may be gathered from the spatial distribution of dSphs, which tend to cluster tightly 
around the giant spirals of the Local Group. This observation is a basic ingredient of dSph formation models, many of which rely on proximity to a giant galaxy in order to shield them from accretion of intergalactic material that might otherwise fuel recent episodes of star formation. Besides depriving them from star formation fuel, proximity to a central galaxy may allow for tidal interactions to sculpt spheroidals out of otherwise irregular galaxies, as in the model championed by Mayer et al (2007, see additional references therein).

One oddity in this context is the presence of two isolated dSphs, Cetus and Tucana, which are found far from both M31 and MW, and orbiting in the outskirts of the Local Group. A possible resolution, however, has been recently argued by Sales et al (2007), who point out that in hierarchical models satellite galaxies often accrete into the primary as systems of multiple objects. The multiple-body interactions involved in the tidal dissolution of these systems may propel some satellites onto highly-energetic orbits that would take them far away from their parent galaxy. More difficult to reconcile with this scenario is the existence of dSphs infalling for the first time into the Local Group, as has been argued for And XII (Martin et al. 2006, Chapman et al. 2007). If correct, this interpretation would imply that at least some dSphs are able to form in a radically different environment well away from giant spirals.

The role of tides in shaping dSphs is nowhere clearer than in the case of the Sagittarius dwarf (Ibata et al 1994), which is in the process of shedding, as a result of its interaction with the Milky Way, a significant fraction of its stars in large "tails" that have been detected all across the sky (Martínez-Delgado et al. 2001, Majewski et al. 2003; Belokurov et al. 2006). Tides are likely to continue to strip Sagittarius in the future, and it is therefore a fair and interesting question to ponder what the eventual fate of Sagittarius will be once it loses most of its stars.

This is a particularly timely issue in light of the newly discovered population of ultra-faint dwarfs around M31 and MW. These systems are roughly 10 to 100 times fainter than the "classical" dSphs introduced above; have amorphous morphology and very low surface brightness (Belokurov et al. 2007, Zucker et al. 2007 and references therein). Could these ultra-faint dwarfs be the tidal remnants of a population of brighter objects now in the process of being tidally stripped into oblivion?

Signs of ongoing tidal disruption would be much harder to detect in systems fainter than Sagittarius (the brightest of all known dSphs), but such a possibility should not be hastily discarded. Indeed, it is only recently that a couple of well-known globular clusters (Pal 5, Odenkirchen et al. 2001, and NGC5466, Belokurov et al. 2006a) have been convincingly shown to exhibit the telltale signatures of tidal stripping. The unambiguous identification of tidally-induced features is even harder when the orbits are eccentric, since in that case the episodes of stripping are short and restricted to pericentric passages. A dwarf is expected to relax back to equilibrium on a dynamical timescale, erasing quickly any signs of the interaction from the main body of the dwarf, where crossing times are shortest.

This difficulty has not deterred a rich body of work from postulating links between tides and the present-day structure of Galactic dSphs. This is typically argued on the basis of the projected stellar density profile, $\Sigma(R)$, where deviations or "breaks" from a smooth profile are often interpreted as evidence for the effect of tides.

Such interpretation is encouraged by the success of King models (King 1966) at reproducing the surface brightness profile of most dSphs. In particular, King models describe well the central region of nearly constant density (the "core") as well as the precipitous decline in the outer regions usually ascribed to a "tidal radius" imposed by the gravitational field of the Galaxy. "Features" in the outer profile, such as an upturn in $\Sigma(R)$ relative to the best-fitting King model, are therefore interpreted as caused by "unbound" stars leaving the dwarf.

The latter assertion, however, is quite difficult to distinguish from more prosaic interpretations where the observed complexities in $\Sigma(R)$ are ascribed to radiallydependent variations in star formation history: in other words, a dSph may deviate from a King model simply because it was born like that. The recent discovery of distinct (bound) dynamical populations in some Local Group dSphs (Sculptor, Tolstoy et al. 2004; Canes Venatici I, Ibata et al. 2006; Fornax, Batagglia et al. 2007; Andromeda II, McConnachie, Arimoto \& Irwin 2007), and the breaks in $\Sigma(R)$ that such populations can produce (see, e.g., Figure 2 of McConnachie, Peñarrubia \& Navarro 2007) suggest that this alternative explanation should be taken into consideration.

Although it might be difficult to determine conclusively whether the properties of a dSph have been modified by the action of tidal forces, it is clear that at least in some cases, like Sagittarius, tides are at work and will determine the dwarf's ultimate structure. This brings about interesting questions. One relates to the link between tides and the "King-like" $\Sigma(R)$ profile of many dSphs. How resilient is $\Sigma(R)$ to the effect of tides? Would a dSph where stars initially follow a King-like model evolve away from it, approaching, for example, a power-law profile as it soaks gravitational energy from tides? Can this be used to gauge the dynamical importance of tides in the evolution of a dSph?

We explore these issues here using cosmologicallymotivated models for dSphs. These models are similar to those introduced by Peñarrubia, McConnachie \& Navarro (2007, hereafter PMN, see also Strigari et al 2007), who used them to constrain the dark matter halos of Local Group dSphs under the assumption that tides have not perturbed drastically the halo properties. This approximation, however, is unlikely to hold for many dSphs and therefore it is imperative to examine the effects of tides on such estimates.

Following PMN, we assume that dSphs are dark matter-dominated systems where stars are "Kingmodel"-distributed tracers embedded within a cold dark matter (CDM) halo. These systems are on eccentric orbits that bring them periodically close enough to the center of the Galactic potential for tides to act. We explore a wide range of orbital parameters, as well as the dependence of our results on the degree of segregation of the stellar component within the dark halo. Our analysis focuses on the equilibrium structure of the remnants, and are thus best applied to systems near the apocenter of their orbits and away from regions where tides are strongest. We will address in a separate contribution 
(Peñarrubia et al., in prep.) the issue of transient departures from equilibrium and their use as diagnostics of the importance of tides.

One limitation is that our dSph models are evolved individually within a rigid Galactic potential, and therefore our computations neglect the effects of dynamical friction (e.g. Peñarrubia et al. 2002, 2004, 2006) and of interactions between satellites (Peñarrubia \& Benson 2005). Since we concentrate on some of the faintest (and thus presumably least massive) systems known, these omissions should not prove deleterious to our conclusions.

This paper is organized as follows. In Sec. 2 we introduce our dSph modeling and describe in detail the numerical technique. Sec. 3 presents our main results regarding the dynamical evolution of dSphs driven by tides. In Sec. 4 we apply our results to the population of Local Group dSphs and newly-discovered ultra-faint galaxies. We end with a brief summary in Sec. 5 .

\section{NUMERICAL MODELING}

Our model assumes that the stellar component of dSphs follow a King (1966) model embedded within a CDM halo. The dark halo is modeled as an N-body realization of a spherical Navarro, Frenk \& White (1996, 1997) (hereafter NFW) profile. Stars are assumed to contribute negligibly to the potential, and may therefore be followed by assigning an energy-dependent "mass-tolight ratio" to each dark matter particle in the equilibrium halo. The dwarf is assumed to orbit within a massive host system which we model, for simplicity, also as a rigid NFW potential.

Our approach is thus similar to that of Hayashi et al (2003), Bullock \& Johnston (2005), and Kazantzidis et al (2004), but we concentrate the analysis on the evolution of the "stellar component" of the main body of the dwarf. Further numerical details are given in the remainder of this section.

\subsection{The host galaxy potential}

The host galaxy potential follows from the NFW density profile, which may be expressed as,

$$
\rho_{\mathrm{NFW}}(r)=\frac{M_{\mathrm{vir}}}{4 \pi r_{s}^{3}} \frac{\left(r / r_{s}\right)^{-1}\left(1+r / r_{s}\right)^{-2}}{\ln (1+c)-c /(1+c)},
$$

where $M_{\text {vir }}$ is the mass within the virial radius, $r_{\text {vir }}, r_{s}$ is a scale radius and $c$ is the NFW concentration parameter, defined as $c \equiv r_{\text {vir }} / r_{s}$.

The virial radius is defined so that the mean overdensity relative to the critical density is $\Delta$,

$$
\frac{M_{\mathrm{vir}}}{(4 / 3) \pi r_{\mathrm{vir}}^{3}}=\Delta \rho_{\text {crit }},
$$

where $\rho_{\text {crit }}=3 H_{0}^{2} / 8 \pi G$, and $H_{0}=100 h \mathrm{~km} / \mathrm{s} / \mathrm{Mpc}$ is the present day value of Hubble's constant.

The choice of $\Delta$ varies in the literature, with some authors using a fixed value, such as NFW, who adopted $\Delta=200$, and others who choose a value motivated by the spherical collapse model, where (for a flat universe) $\Delta \sim 178 \Omega_{\mathrm{m}}^{0.45}$ (Lahav et al 1991, Eke et al 1996). The latter gives $\Delta=95.4$ at $z=0$ in the concordance $\Lambda \mathrm{CDM}$ cosmogony, which adopts the following cosmological parameters: $\Omega_{\mathrm{m}}=0.3, \Omega_{\Lambda}=0.7, h=0.7$, consistent with

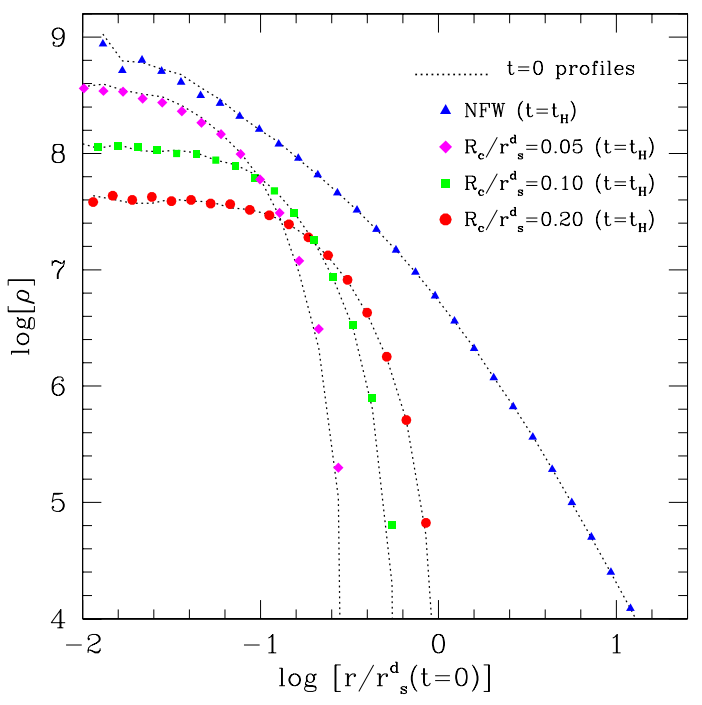

FIG. 1.- Density profiles of the King-NFW dwarf galaxy model considered in this paper (arbitrary units). The dark halo is described as an NFW model (top curve); the stellar component is assumed to follow a King model constructing by sampling the NFW model in energy space (other lines). Three different choices for the spatial segregation between stars and dark matter are shown: $R_{c} / r_{s}=0.05,0.1$, and 0.2 . Dotted lines correspond to the models as initialized by the N-body procedure. Symbols show the configuration of the same models, but after evolving the system for 14 Gyr in isolation. The good agreement between lines and symbols indicate that the dSph model is in equilibrium and free from numerical artifact induced by numerical limitations down to scales comparable to the grid size of the highest resolution zone used in SUPERBOX, $r_{s} / 126$.

constraints from CMB measurements and galaxy clustering (see, e.g., Spergel et al 2006 and references therein).

Our models are purely gravitational and therefore scale-free. When convenient for interpretive purposes, we shall scale our numerical units to physical parameters assuming that the host galaxy potential has $M_{\mathrm{vir}}=$ $7.55 \times 10^{11} M_{\odot}, r_{\text {vir }}=233.0 \mathrm{kpc}$ and $r_{s}=8.18 \mathrm{kpc}$, assuming $\Delta=101.1$. This corresponds to a virial velocity of $V_{\text {vir }}=117.8 \mathrm{~km} / \mathrm{s}$ and a circular velocity profile that reaches its peak, $V_{\max }=188.1 \mathrm{~km} / \mathrm{s}$, at $r_{\max } \approx 2 r_{s}=16.4 \mathrm{kpc}$. An NFW profile is fully characterized by a pair of independent parameters, for example, $M_{\text {vir }}$ and $c$, or $r_{\max }$ and $V_{\max }$. by

The potential corresponding to an NFW halo is given

$$
\Phi_{\mathrm{NFW}}(r)=-\frac{M_{\mathrm{vir}}}{r} \frac{\ln \left(1+r / r_{s}\right)}{\ln (1+c)-c /(1+c)},
$$

and is assumed to remain constant during the evolution of the dwarf.

For simplicity, our host galaxy model does not include the presence of a disk or bulge. The presence of these components would break the spherical symmetry of the host halo and increase the number of free parameters needed to describe it in detail. However, as we shall show below, the effects of tides on the structure of dSphs seem to depend solely on the total amount of mass lost to tides, and not on the details of how it was stripped. In this sense, our results are likely to depend largely on the strength of the tides and only weakly on the exact potential shape of the host galaxy, and our assumption 

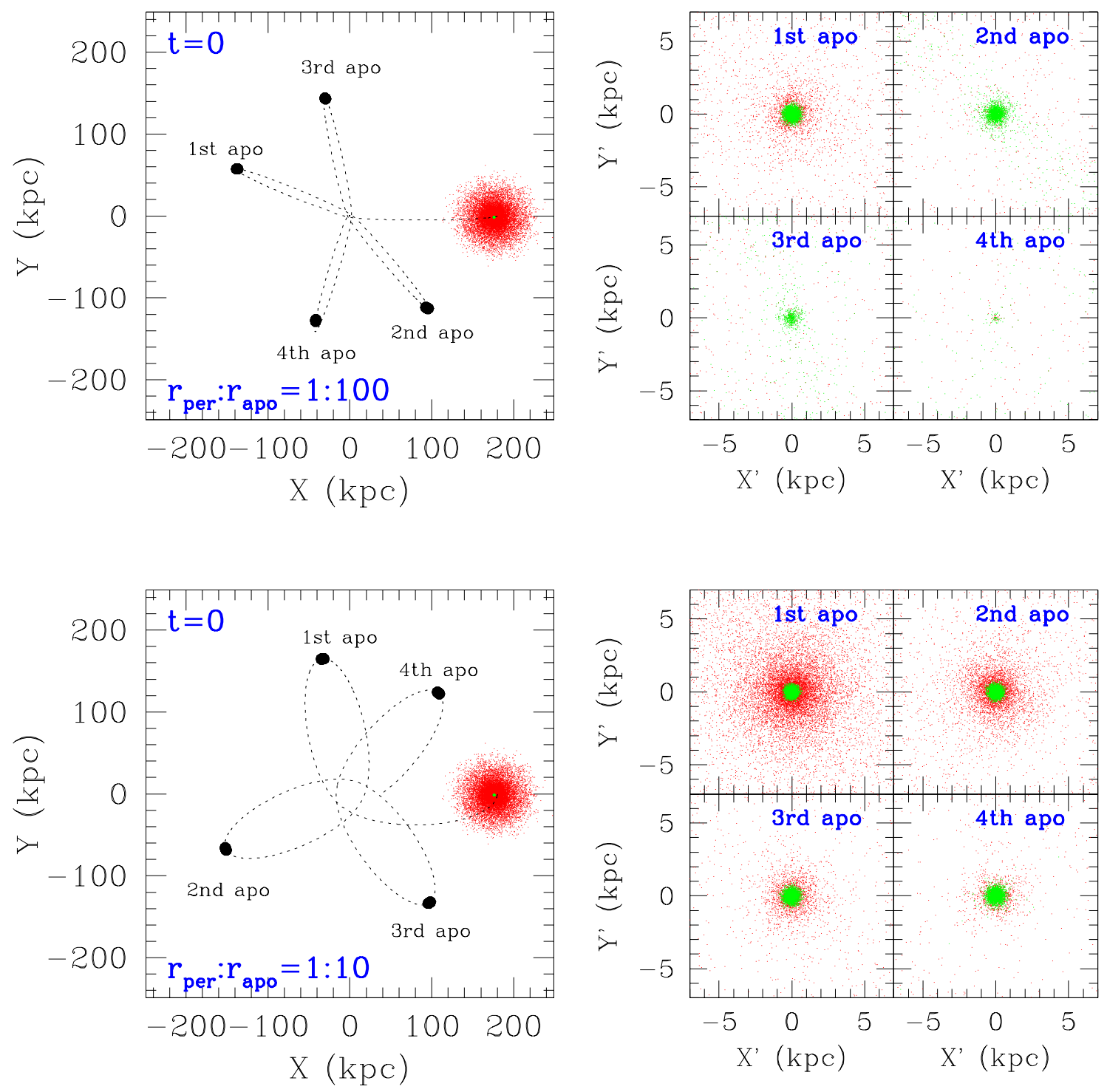

FIG. 2.- Projection onto the orbital plane of two dSph models with different orbital pericenter. Left panels show the initial systems at scale. Right-hand panels show the inner $15 \mathrm{kpc}$ of the dwarf at various times corresponding to four consecutive apocenters for each orbit. Red and green dots denote, respectively, dark and stellar particles. Note how the stripping proceeds from the outside in and that a large fraction of the dark matter halo must be tidally stripped before stellar mass loss begins.

of a spherically symmetric halo should still capture the most relevant features of this process.

\subsection{The dSph $N$-body realization}

Our dwarf galaxy N-body model is constructed by generating an equilibrium 5 million-particle realization of the NFW profile, using a code kindly made available by S.Kazantzidis (Kazantzidis et al. 2004, 2006). Since the NFW profile has infinite radial extent and divergent total mass, the profile is truncated outside the virial radius.

We have chosen parameters for the dwarf consistent with those expected for dSph halos (see, e.g., PMN). Using the same physical scaling adopted for the host galaxy $\left(\S\right.$ [2.1) we adopt $M_{\text {vir }}=3.7 \times 10^{9} M_{\odot}, r_{\text {vir }}=39.6 \mathrm{kpc}$, and $c=20$. This implies $V_{\text {vir }}=20.0 \mathrm{~km} / \mathrm{s} ; r_{s}=2.0 \mathrm{kpc}$; $V_{\max }=28.6 \mathrm{~km} / \mathrm{s}$; and $r_{\max }=4.0 \mathrm{kpc}$.

We emphasize, however, that our models are scale free, so that the only relevant parameters in the cal- culations are the relative masses and sizes of the host and dwarf, which may be expressed by the ratio of their maximum circular velocities, $V_{\max }^{\text {host }} / V_{\max }^{\mathrm{dSph}}=6.6$ and by $r_{\max }^{\text {host }} / r_{\max }^{\mathrm{dSph}}=4.1$. In what follows, a "d" or " $\mathrm{h}$ " upper script will be used to denote quantities corresponding to the "dwarf" or the "host" models, respectively.

Our model assumes that stars are mass-less tracers of the potential, and therefore we track the stellar component using a subset of dark matter particles, chosen so that they describe a King model in equilibrium within the dwarf halo. We follow the method outlined by Bullock \& Johnston (2005), and assign weights to dark matter particles so as to select $f_{K}(\epsilon) / f_{\mathrm{NFW}}(\epsilon) \times N(\epsilon)$ stars from each bin in relative energy, $\epsilon \pm \delta \epsilon$. Here $f_{K}$ and $f_{\mathrm{NFW}}$ are the isotropic distribution functions for the stellar and 


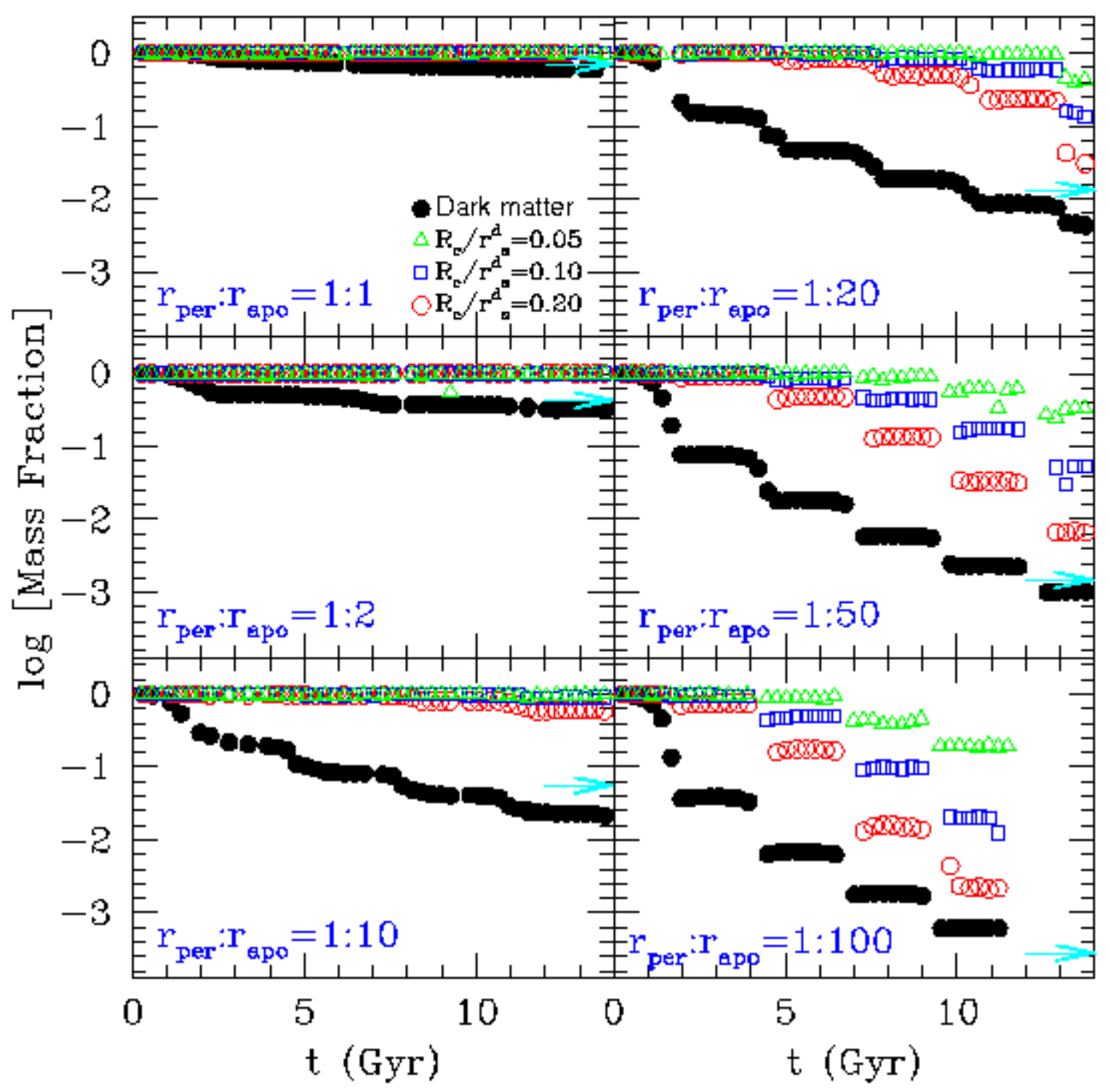

FIG. 3.- Bound mass fraction as a function of time for different orbits and stellar segregations. Arrows indicate the mass fraction within the theoretical "tidal radius" estimated at pericenter (see text for details). Note that a large fraction of the halo mass must be tidally stripped $(\sim 90 \%)$ before stars begin to be stripped. However, when stars are stripped, they are lost more efficiently than the remainder of the dark halo. See text for details.

the dark matter particles calculated according to

$$
f_{i}(\epsilon)=\frac{1}{8 \pi^{2}}\left[\int_{0}^{\epsilon} \frac{d^{2} \rho_{i}}{d \Psi^{2}} \frac{d \psi}{\sqrt{\epsilon-\Psi}}+\frac{1}{\sqrt{\epsilon}}\left(\frac{d \rho_{i}}{d \Psi}\right)_{\Psi=0}\right],
$$

where the subscript $i$ denotes that $\rho$ can be either an NFW or a King profile, and $\Psi$ is the total gravitational potential.

The result is a set of $N_{\star}$ dark matter particles that follow a King (1962) density distribution,

$\rho_{\star}=\frac{K}{x^{2}}\left[\frac{\cos ^{-1}(x)}{x}-\sqrt{1-x^{2}}\right], \quad x \equiv\left[\frac{1+\left(r / r_{K}\right)^{2}}{1+\left(r / r_{t}\right)^{2}}\right]^{1 / 2}$

where $r_{K}$ and $r_{t}$ are the King and tidal radii and $K$ is an arbitrary constant. For all our dSph models we have fixed $r_{t} / r_{K}=5$.

To compare with observations it is easier to use the projected King (1962) surface density

$$
\Sigma(R)=k\left\{\frac{1}{\left[1+\left(R / R_{c}\right)^{2}\right]^{1 / 2}}-\frac{1}{\left[1+\left(R_{t} / R_{c}\right)^{2}\right]^{1 / 2}}\right\}^{2}(6)
$$

where $R$ is the projected radius and $k$ an arbitrary constant. For our choice of $r_{t} / r_{K}=5$, we have that our King models have "concentration" $c_{K} \equiv R_{t} / R_{c}=5$, and central surface brightness given by $\Sigma_{0}=k\left(1-1 / \sqrt{1+c_{K}^{2}}\right)^{2}$.

With these choices, the whole procedure depends solely on the degree of spatial segregation between stars and dark matter in the dwarf, which we may express as the ratio between the stars' core radius and the halo NFW scale radius, $R_{c} / r_{s}^{\mathrm{d}}$. As discussed by PMN, this segregation parameter may be constrained by using the cosmological relationships between the mass and concentration of NFW halos obtained from N-body simulations 


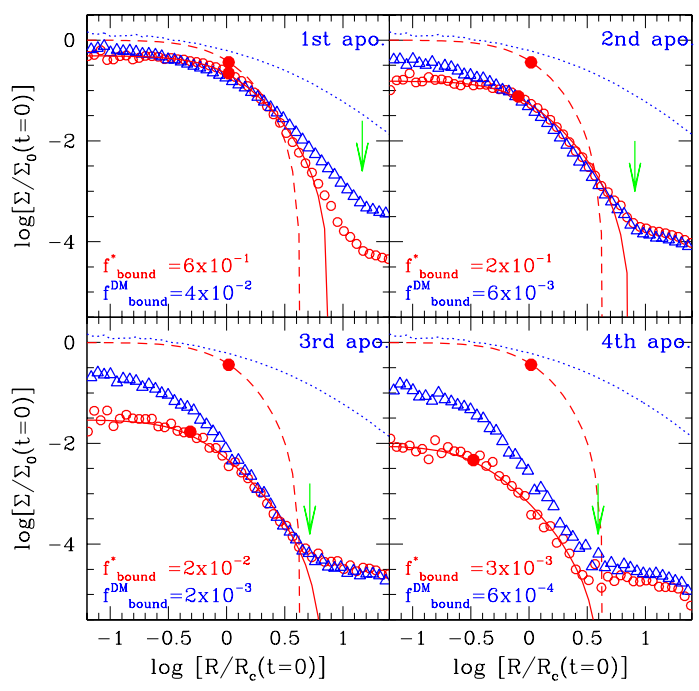

FIG. 4.- Surface density profiles of our dSph model (assuming a stellar segregation of $\left.R_{c} / r_{s}^{d}=0.20\right)$ moving on a highly eccentric orbit $\left(r_{\text {per }}: r_{\text {apo }}=1: 100\right)$ at different orbital apocenters. Stellar and dark matter profiles are denoted with open circles and triangles, respectively. For comparison we also plot the initial profiles in each panel (dotted lines). At each snapshot we fit a King profile to the stellar component (solid lines). Solid dots show the resulting value of the core radius $\left(R_{c}\right)$. Arrows indicate the position of $R_{\text {eq }}$, where the crossing time equals the time elapsed since pericenter. Labels in each panel indicate the remaining fraction of bound stellar and dark mass, respectively. See text for details.

(e.g. NFW, Eke, Navarro \& Steinmetz 2001, Bullock et al 2001). PMN find values of $R_{c} / r_{s}^{d}$ in the range $0.05-0.20$ (or, equivalently, $R_{c} / r_{\max }^{d} \simeq 0.025-0.1$ ), which implies that the stellar King models are deeply embedded within their NFW halos. This results in velocity dispersion profiles, $\sigma_{p}(R)$, that, in concordance with observations, are nearly flat almost out to the "tidal" radius.

The more deeply segregated the stellar component is, the fewer particles are available to trace it, and therefore large numbers of particles are required in order to resolve the innermost regions of halos where the stars are thought to reside. Using a total number of $5 \times 10^{6}$ dark matter particles for the NFW profile we obtain stellar components traced by $1.95 \times 10^{4}, 6.86 \times 10^{4}$, and $1.88 \times 10^{5}$ particles, for $R_{c} / r_{s}=0.05,0.10$, and 0.2 , respectively.

\subsection{The N-body code}

We follow the evolution of the dSph N-body model in the host potential using SuPERBOx, a highly efficient particle-mesh gravity code (see Fellhauer et al. 2000 for details). SupERBox uses a combination of different spatial grids in order to enhance the numerical resolution of the calculation in the regions of interest. In our case, SuPERBOX uses three nested grid zones centered on the highest-density particle cell of the dwarf. This center is updated at every time step, so that all grids follow the satellite galaxy along its orbit.

Each grid has $128^{3}$ cubic cells: (i) the inner grid has a spacing of $d x=r_{s}^{d} / 126 \simeq 8 \times 10^{-3} r_{s}^{d}$ and is meant to resolve the innermost region of the dwarf. As discussed above, we assume that stars are heavily segregated relative to the dark matter, so that the inner grid spacing is of order $d x \simeq R_{c} / 6-R_{c} / 25$. (ii) The middle grid extends to cover the whole dwarf, with spacing $r_{\text {vir }}^{d} / 126$. (iii) The outermost grid extends out to $50 \times r_{\text {vir }}^{d}$ and is meant to follow particles that are stripped from the dwarf and that orbit within the main galaxy..

SuPERBOX uses a leap-frog scheme with a constant time-step to integrate the equations of motion for each particle. We select the time-step according to the criterion of Power et al (2003); applied to our dwarf galaxy models, this yields $\Delta t=4.6$ Myr for the physical unit scaling adopted in Sec. 2.1.

\subsection{Tests of the dSph model}

We have checked explicitly that our procedure for building the dSph model leads to a system that is in equilibrium and that does not evolve in isolation away from the prescribed configuration. This is shown in Fig. 1 , where we show the density profile of the NFW profile (top dotted line), as well as the profile of the King-model subsets, for three choices of $R_{c} / r_{s}$ (other lines, arbitrary vertical normalization). The symbols show the same profiles, but after evolving the system in isolation for $14 \mathrm{Gyr}$ with Superbox. The good agreement between lines and symbols shows that our numerical choices are appropriate, and that the evolution of the N-body model, on the scales of interest, is free from artifact induced by the finite spatial and time resolution of the calculation.

\section{TIDAL EVOLUTION OF DWARF SPHEROIDALS}

\subsection{The Orbits}

In order to explore the tidal evolution of dSphs we have placed the dwarf galaxy model in orbit within the potential of the host galaxy assuming 6 different combinations of apocentric/pericentric ratio. All 6 orbits have apocenters $r_{\text {apo }}=0.77 r_{\text {vir }}^{\mathrm{h}}=180 \mathrm{kpc}$. The strength of the tidal forcing is thus controlled by the pericentric radius, which we vary from $r_{\text {per }}=r_{\text {apo }}$ (a circular orbit) to $1 / 2,1 / 10$, $1 / 20,1 / 50$, and $1 / 100$ of $r_{\text {apo }}$. Scaling this to our chosen physical units, the orbit with the smallest pericenter reaches within $\sim 2 \mathrm{kpc}$ of the center of the host, but one should bear in mind that the scaling is arbitrary, and that it is best to regard the series of simulations as one of increasing tidal strength for eccentric orbits of similar orbital period.

We may quantify the expected strength of the tides by computing the theoretical "tidal radius" of the dwarf galaxy model if it were placed on a circular orbit at pericenter. Defining $r_{\text {tid }}$ as $\left\langle\rho^{\mathrm{d}}\right\rangle\left(r_{\text {tid }}\right)=3\left\langle\rho^{\mathrm{h}}\right\rangle\left(r_{\text {per }}\right)$ (where $\langle\rho\rangle(r)$ denotes mean enclosed density within $r)$ we may estimate the total amount of mass, $M_{\text {tid }} \equiv M^{d}\left(r_{\text {tid }}\right)$, that the satellite might be expected to retain after completing several orbits in the host potential.

The results are compiled in Table 1. Except for the circular orbit case, it is clear from inspecting these values that we expect tides to have a strong influence on the evolution of the dwarf. For example, more than half of the initial mass is expected to be lost in the $r_{\text {per }}: r_{\text {apo }}=1: 2$ orbit, growing to $99.97 \%$ in the case of the most extreme orbit (1:100). In all cases we follow the evolution of the satellite for about $t_{H}=14$ Gyr (in our scaled units), which corresponds to roughly $3-5$ radial orbital periods.

We show for illustration the orbital paths of two simulations in the left-hand panels of Fig. 2. These correspond to the $r_{\text {per }}: r_{\text {apo }}=1: 100$ and $1: 10$ cases, respec- 


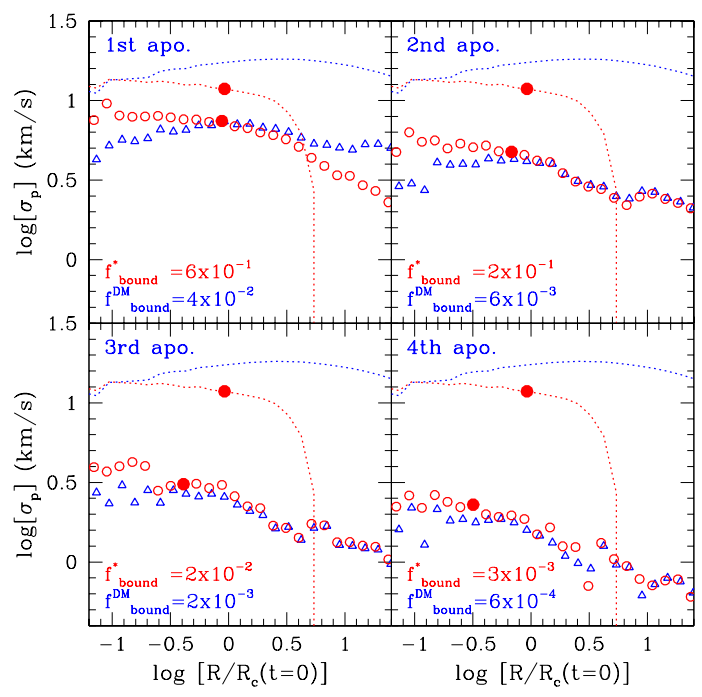

Fig. 5.- As Fig. 4 but for the projected velocity dispersion profiles. Only bound particles are considered in these panels. Note that tidal stripping leads to a monotonic decrease of $\sigma_{p}(R)$ at all radii. However, even after extreme mass loss, the shape of the velocity dispersion profile of the bound stellar remnants remains approximately constant out to roughly $\sim 3 R_{c}$.

tively. The dark matter particles of the dSph model are shown in red, and are at scale. The stellar component is shown in green (assuming $R_{c} / r_{s}^{\mathrm{d}}=0.2$ ), and is barely seen as a dot at the center of the dwarf; this gives a visually intuitive impression of how deeply segregated the stars are relative to the dark matter in our models.

\subsection{Mass loss}

As expected, tides are strongest at pericenter and trigger recurring episodes of mass loss at every pericentric passage. (The obvious exception is the circular orbit case, where little stellar mass loss is expected, see Table11) The progressive stripping of the satellite is shown in Fig. 3, where the solid circles show the fraction of mass that remains bound to the satellite as a function of time. The pericentric passages are easily recognizable in this plot as the times when sudden drops in bound mass occur. Except at pericenter, the bound mass of a satellite remains fairly constant during the orbit, suggesting, as indicated above, that tides act nearly impulsively along the chosen orbits and have little influence on the main body of the dSph at times other than pericenter.

The inner regions of the halo are remarkably resilient to disruption by such "tidal shocks". Indeed, after 14 Gyr all of our dSph models, except for the most extreme orbit, still retain a fraction of their mass. The retained mass fraction after completing 3-5 orbits is in reasonable agreement with the initial mass within the "tidal radius", $r_{\text {tid }}$, which is shown by the rightward pointing arrows in Fig. 3 Despite this coincidence, there is no sign that the retained mass is converging to a well defined value in the case of the three most eccentric orbits. The dSph halo is fully disrupted during the 5 th pericentric passage of the 1:100 orbit although one would have expected that $\sim 0.03 \%$ (about 2000 particles) should remain bound in this case. A similar result is obtained for the 1:50 orbit when extending the simulation beyond the nominal 14 Gyr timespan. This suggests that the "tidal mass" listed in Table1 1 actually underestimates the mass that remains attached to a dSph after repeated pericentric passages.

Mass loss loss affects predominantly the outer regions of the dSph halo. This is shown by the open symbols in Fig. 3, which indicate the evolution of the stellar bound mass fraction, for three different values of the segregation parameter; $R_{c} / r_{s}^{d}=0.05,0.1$, and 0.2 . The stellar mass loss, as a whole, is always less pronounced than the dark matter's, as expected for a stellar system that is deeply embedded within its dark halo. Depending on how segregated the stars are, a dSph may lose $\sim 90 \%$ of its original dark mass and still retain all of its stars (see, e.g., the bottom left panel in Fig. 3). For the range of segregations adopted here, a dSph must lose at least 90\% of its mass before starting to shed its stars.

The outside-in "onion-peel" stripping of the dSph may be appreciated in the right-hand panels of Fig. 2, where we show, at consecutive apocenters of the orbit, the distribution of dark matter particles (in red) and stars (in green) in a $15 \mathrm{kpc}$ box centered on the dwarf. This shows clearly how a halo is gradually stripped of its mass from the outside in. The bottom panels of this figure, in particular, illustrate how a dSph may be stripped of much of its halo whilst leaving most of its stars bound. The stellar components of dwarf halos are clearly extremely resilient to tidal stripping.

Finally, another interesting result may be gleaned from Fig. 3 by noting that, when stars are lost, the "jumps" in bound stellar mass are larger than those associated with the dark matter as a whole. This implies that, when the stellar component actually begins to disrupt, it does so more rapidly than the remainder of its dark matter halo. This has interesting consequences for the evolution and interpretation of the dSph structural parameters, an issue to which we return below.

\subsection{Evolution of the density profile}

Fig. 4] shows the evolution of the projected density profile of dark matter (triangles) and stars (circles) for the most extreme orbit $\left(r_{\mathrm{per}}: r_{\mathrm{apo}}=1: 100\right)$ probed in our series. The stellar profile is shown for the case when the segregation parameter is $R_{c} / r_{s}^{d}=0.2$. The system is shown at four consecutive apocentric passages, when the main body of the dSph has had some time to relax after being acted on by tides at pericenter. The dotted lines are the same in each panel, and show the initial dark matter and stellar profiles, for ease of reference. The vertical normalization of the initial stellar profile is arbitrary, but is the same in all panels.

This figure illustrates a few interesting points. One is that it is possible to identify two distinct regions on the basis of the shape of the density profile: an inner region where the profile varies smoothly with radius, and an outer region where an "excess" of mass is present relative to a naive extrapolation of the inner profile. The transition radius coincides roughly with the arrow marking the distance from the center where the crossing time equals the time elapsed since pericenter, $t-t_{\mathrm{per}}=t_{\text {cross }}\left(R_{\mathrm{eq}}\right)=R_{\mathrm{eq}} / \sigma_{0}$, where $\sigma_{0} \equiv \sigma_{p}(R=0)$ is the central line-of-sight stellar velocity dispersion.

Particles inside $R_{\mathrm{eq}}$ are thus within the region where enough time has elapsed for equilibrium to be reestablished, whereas the outer region still contains a large number of weakly-bound particles that are still moving 
out and have yet to settle into their new orbits (Aguilar \& White 1985, 1986, Navarro 1990). These particles are responsible for the transient "excess" of mass in the outer regions. The location of this excess moves gradually outward, and it would therefore be difficult to detect unless a dwarf is inspected soon after pericenter. Note that stars in the outer envelope are not necessarily unbound, and that the presence of this "tidal break", as the outer excess is sometimes referred to, does not necessarily imply the presence of an unbound population.

A second point of note is that, within $R_{\text {eq }}$, the stellar density profile is very well approximated by a King model, as shown by the solid line fits to the inner open circles in Fig. 4 . The solid dot indicates the core radius of the fit; intriguingly, this seems to evolve rather weakly, changing by less than a factor of $\sim 3$ even when the stellar component has been stripped of roughly $99.7 \%$ of its original mass. Stellar mass loss thus correlates almost inversely proportional to the central surface density, which drops by about two decades after completing 4 orbits.

The change in projected density is much less pronounced in the dark matter, emphasizing that, when stars are lost to tides, they do so with higher efficiency than the remaining dark matter. This is because the dark matter profile is "cuspy" whereas that of the stars is "cored": the dark matter in the very inner regions is therefore more resilient to tides than stars. As a result, tides lead generally to an increase in the mass-to-light ratio of a dSph remnant. This statement depends critically on the cuspy inner profile of the dark matter, and may therefore be regarded as a genuine prediction of the $\Lambda$ CDM scenario.

\subsection{Evolution of the velocity dispersion profile}

The evolution of the velocity dispersion profile of stars and dark matter is shown in Fig. 5. As in Fig. 4, the dotted lines indicate the initial profiles and are the same in all panels. The solid dot marks the core radius of the best King model fits to the equilibrium region, i.e., $R<R_{\text {eq. }}$. Unlike the plots in Fig 4, we use only bound particles to compute the velocity dispersion. As discussed in the literature, the presence of unbound stars would increase the velocity dispersion estimates and may lead to "features" in the velocity dispersion profile. Observationally, the interpretation of these features is difficult, since such features may be produced by stars stripped from the dwarf or by unrelated objects projected onto the line of sight (e.g. Johnston et al. 2002, Read et al. 2006, Fellhauer \& Kroupa 2006, Klimentowski et al. 2007). However, considering only bound particles, the velocity dispersion profiles show no obvious feature separating the equilibrium region from the unrelaxed envelope. Thus, the profiles shown in Fig. 4 would be expected in galaxies that have had time to relax after the pericentric passage and whose unbound component has already evacuated the progenitor system.

For Figures 4 and [5] we have selected a relatively extended stellar component $\left(R_{c} / r_{s}^{d}=0.2\right)$ to show the enhanced effects of tidal interactions. This results in an initial velocity dispersion profile that is slightly declining at large radii. Measurements of $\sigma_{p}(R)$ in the Local Group dSphs show nearly flat velocity dispersion profiles within at least $3 R_{c}$ (see $\left.\S 1\right)$. As discussed by PMN, that condition implies $R_{c} / r_{s}^{d} \lesssim 0.1$ for our initial models.
Remarkably, Fig. 5 shows that tidal mass stripping does not alter the velocity dispersion profile of the bound stellar component, even after the dwarf galaxy loses $99 \%$ of the original stars: at $R \sim 3 R_{c}$ drops by less than $40 \%$ from its central value in all four panels of this Figure. The central velocity dispersion, on the other hand, drops by a factor of $\sim 5$ after the system has lost $99.7 \%$ of its stars, a result that will prove useful when trying to interpret observationally the tidal remnants of Local Group dSphs.

Note that tides preserve the "King-like" structure of the stellar component and that, therefore, the bound remnants of stripped dwarf galaxies will show no obvious signature of tidal effects once they have been able to relax to virial equilibrium after the latest pericentric passage.

\subsection{Evolution of stellar structural parameters}

As shown in Fig. 4. NFW-embedded King models are very resilient to the effect of tides; even after being stripped of $99 \%$ of its stars, the remaining bound stellar component resettles into a King-like configuration in just one dynamical time. The change in the best fitting King-model parameters will depend on the strength of the tidal interaction as well as on the spatial segregation between stars and dark matter. The more segregated the stellar component the harder it is for tides to affect it.

This is demonstrated in the panels on the left of Figure 6. which show, as a function of time, the evolution of the central surface density, $\Sigma_{0}$, core radius, $R_{c}$, and King "concentration", $c_{\mathrm{k}}=R_{t} / R_{c}$, for three of the six orbits surveyed in our study. Different symbols correspond to different orbits, and are shown at consecutive orbital apocenters, when the main body of the dSph is close to equilibrium. Open and filled symbols correspond to assuming a radial segregation between stars and dark matter of $R_{c} / r_{s}^{d}=0.1$ and 0.2 , respectively. As shown in Fig. 4, these fits are good over one to two decades in surface density, a range comparable to that normally used in observations of Local Group dSphs. The panels in Figure 6] also include the central line-of-sight velocity dispersion, $\sigma_{0}$, measured using stars within one core radius of the center.

The main evolutionary trends driven by tides are well defined. Tidal effects lower the surface brightness; reduce the core radii; increase the King concentration; and reduce the velocity dispersion of stars embedded within NFW halos.

These panels also make clear that the results are highly dependent on (i) the pericenter of the orbit; (ii) the number of orbital periods completed; as well as (iii) the radial segregation between stars and dark matter. The individual impact of these parameters is highly degenerate, so that a particular modification in the dSph structure may be achieved by trading off the effect of one against another. A certain amount of mass loss, for example, may be achieved by completing several orbits of moderate pericenter, or by completing a single one with smaller $r_{\text {per. }}$.

Interestingly, the degeneracy between these various effects can be collapsed into a single parameter, which may be used to describe the structural changes in the stellar component fairly accurately. Such parameter may be expressed, for example, as the total mass within the 
(initial) core radius of the unperturbed King model, $M_{c} \equiv M\left[r<R_{c}(t=0)\right]$. This is shown in the panels on the right of Fig. 6 irrespective of pericenter, segregation, or number of completed orbits, the evolution of the structural parameters depends solely on how much (and not how) the total mass within the initial core radius has varied.

This is a remarkable result, especially because our series includes extreme cases where the mass within the core radius has decreased by up to a factor of $\sim 30$. Such models have lost $99.7 \%$ of their stars; dropped in surface density by a factor of $\sim 100$; lowered their velocity dispersion by a factor of $\sim 4$; and trimmed their core radii by a factor of $\sim 3$.

We have fitted a simple empirical formula to the evolution of the structural parameters,

$$
g(x)=\frac{2^{\alpha} x^{\beta}}{(1+x)^{\alpha}},
$$

where $x \equiv M_{c} / M_{c}(t=0)$ and $g(x)$ represents any of the quantities plotted in Fig. 6. The best fit values of $\alpha$ and $\beta$ for each case are listed in Table 2, and the results are shown by the dashed lines in Fig. 6 .

\subsection{Evolution of the mass-to-light ratio}

One interesting application of the results presented above is to the evolution of the mass-to-light ratio of a dSph, as inferred from the velocity dispersion and King-model fit parameters. This is traditionally estimated assuming that "mass follows light" as $\Upsilon_{c} \simeq$ $1.447 \sigma_{0}^{2} /\left(\Sigma_{0} R_{h}\right)$ (Richstone \& Tremaine 1986). Here $R_{h}$ is the half-light radius, which may be easily computed for a King model from $R_{c}$ and $c_{K}$.

The result is illustrated in the bottom panel of Fig. 7. where the starred symbols show $\Upsilon_{c}$ as a function of the total luminosity of the bound stellar component (in units of the initial value). This is in good agreement with the evolution of the "true" mass-to-light ratio within the half-light radius $\Upsilon=2 M^{d}\left(<R_{h}\right) / L$, which is indicated by the solid symbols in the same panel. Two different regimes may be discerned here. One corresponds to decreasing mass-to-light ratio when stellar mass loss is moderate: dSphs that have lost less than $2 / 3$ of their original stellar mass see their mass-to-light ratios decrease slightly.

This is because tides actually strip mass from all radii at all times. Indeed, the mass in the inner regions decrease initially as tides strip particles on elongated orbits which, although they spend most of their time at large radii, they still contribute to the total mass in the inner regions. These are preferentially dark matter particles, since stars are (by construction) confined to small radii by the presence of an outer cutoff in the stellar distribution (i.e., the "tidal radius" of the initial King model). As a result, for moderate stellar mass loss more dark matter than stars are lost from within $R_{h}$, and $\Upsilon$ decreases. The effect is not large, however, and $\Upsilon$ never decreases by more than $\sim 40 \%$.

On the other hand, when stars are stripped in earnest the trend is reversed; no dark matter is left outside the outer cutoff in the stellar component, and most particles are stripped from the luminous body of the dwarf. Since the inner dark matter profile is "cuspy" and thus more centrally concentrated than the stars, it is now stars that are preferentially lost, and the mass-to-light ratio, $\Upsilon$, increases. This trend dominates the late stages of stripping: dwarfs that have lost more than $\sim 90 \%$ of their original stars are expected to have higher mass-to-light ratios than unperturbed systems. A system that has retained only $1 \%$ of its stars would have a mass-to-light ratio almost a factor of $\sim 10$ higher than its initial value. We shall use these results below to assess the possible presence of tidal remnants in the Local Group amongst the newly discovered ultra-faint dwarfs.

\subsection{Evolution of the dark halo}

As discussed by PMN, the structural parameters of the stellar component of dSphs may be used to estimate the total mass of their surrounding dark matter halos. These authors show how $\Sigma_{0}$ and $R_{c}$ may be used to constrain, under plausible assumptions, the physical parameters of the surrounding NFW halo, such as $r_{\max }$ and $V_{\max }$. The procedure outlined by PMN, however, assumes that halos are well described by an unperturbed NFW profile, an assumption that clearly breaks down when the halo has been substantially stripped by tides (see, e.g., Hayashi et al 2003, Kazantzidis et al 2004). How severely are the estimates of PMN affected when tides have been at work?

In order to address this question, we explore first how the parameters $r_{\max }$ and $V_{\max }$ describing the halo evolve as it is stripped. This is shown in Fig. 8, and indicates, in agreement with the earlier results of Hayashi et al (2003), that the position of the circular velocity peak is more significantly affected than the peak velocity itself. The symbols in Fig. 8 show the results for all our orbits (measured at every apocenter), and show that halos follow a well defined path in the $r_{\max }-V_{\max }$ plane. In agreement with Hayashi et al (2003), we find that the changes in $r_{\max }$ and $V_{\max }$ depend solely on the total amount of mass lost, and not on the details of how it was stripped. Our most disturbed halo has retained just $0.15 \%$ of its initial mass; its $r_{\max }$ has decreased by a factor of $\sim 30$ and $V_{\max }$ by $\sim 7$.

Note that in the event of large mass loss $r_{\max }$ scales almost linearly with $V_{\max }$, implying that halos evolve in this plane along "tracks" nearly parallel to the cosmological relation linking $r_{\max }$ and $V_{\max }$ of unperturbed $\Lambda \mathrm{CDM}$ halos (denoted roughly by the dotted line in Fig. 8, see NFW or PMN for further details).

As a result, estimating $V_{\max }$ by assuming that the halo is an unperturbed NFW that follows the cosmological relation between $r_{\max }$ and $V_{\max }$ (as in PMN) results at most in a small error. This is shown in Fig. 9, where we show, as a function of stripped stellar mass, the error in $V_{\max }$ incurred by applying the procedure outlined by PMN. Note that even for the most extreme case of tidal stripping probed by our simulations, the resulting error is less than about $\sim 30 \%$. We conclude that the velocity dispersion and core radii of $\mathrm{dSphs}$ may be used to put strong constraints on the maximum circular velocity of its surrounding halo, and that the $V_{\max }$ estimates presented by PMN are relatively insensitive to tidal stripping.

\section{APPLICATION TO LOCAL GROUP DWARFS}



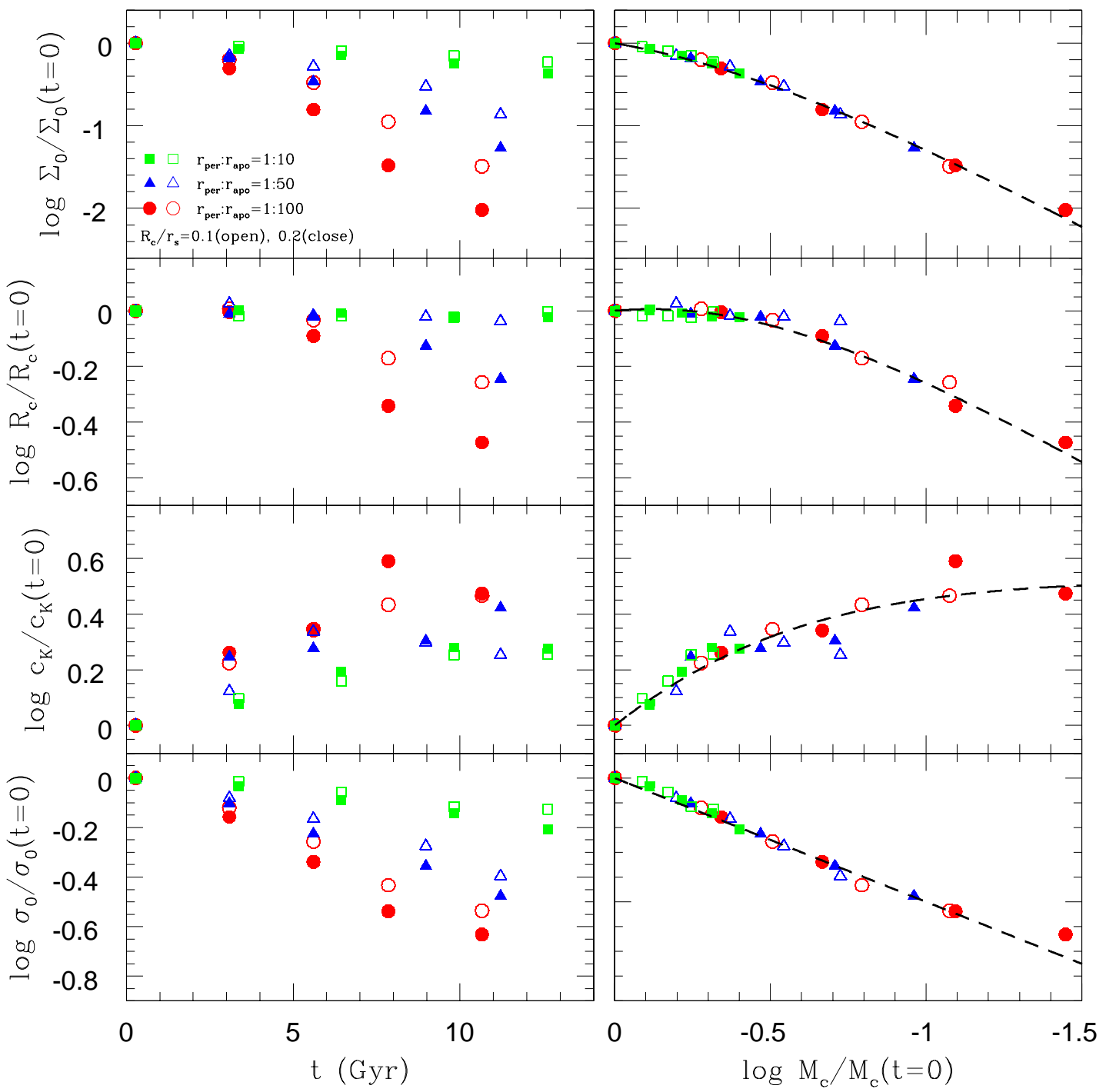

FIG. 6.- Evolution of the parameters of the King models that best fit the inner $\left(R<R_{\text {eq }}\right)$ stellar surface density profile: $\Sigma_{0}, R_{c}$, and $c_{K}=R_{t} / R_{c}$. Also shown is the central projected velocity dispersion, $\sigma_{0}$. These parameters are shown as a function of time (panels on the left) and as a function of the total bound mass within the initial core radius, $M_{c} / M_{c}(t=0)$ (panels on the right). Different symbols correspond to different orbits and stellar segregations measured at consecutive apocenters. Dashed lines in the right-hand panels show our empirical fits (see eq. 7 and Table 2). Note that the evolution of the stellar parameters only depends on the total amount of mass lost from within the luminous radius and not on the details of how this mass was stripped.

The results presented in 93 allow us to assess the effect of tides on the Local Group dSph population and to address some of the questions posed in Sec. 1. We begin by introducing the latest data available for LG dSphs, compiled from the literature, and then discuss the possible role that tides may have played on the structure of these objects.

\subsection{The structural properties of Local Group dSphs}

Table 3 lists the observational parameters most relevant for this discussion, namely the (visual band) luminosity $(L)$, the half-light radius $\left(R_{h}\right.$, measured as the geometric mean of the semi-major and minor axes) and the central velocity dispersion $\left(\sigma_{0}\right)$. The table lists all dSphs discovered so far in the Local Group, including very recent discoveries. They have been divided by environment
(MW satellites, M31 satellites and isolated dSphs) and are listed in order of decreasing luminosity. We distinguish between "classical" dSphs and "ultra-faint" dSphs, as those brighter or fainter than Draco, respectively. Also listed are the peculiar systems Willman 1 and Segue 1, as well as the isolated Cetus dwarf. Finally, Table 3 also lists derived quantities, such as the effective surface brightness $\left(\Sigma_{e}=L /\left(2 \pi R_{h}^{2}\right)\right)$, and the mass-to-light ratio parameter. Since for several of the recently discovered dSphs only estimations of their total luminosity and halflight radius are available, we compute the effective massto-light ratio as $\Upsilon_{e}=5 \sigma_{0}^{2} R_{h} /(G L)$. For a King profile, the relationship between $\Upsilon_{e}$ and $\Upsilon_{c}$ derived by Richstone $\&$ Tremaine (1986) depends upon the value of the concentration $c_{K}$, so that for typical values of $c_{K}=3,5$ and $10, \Upsilon_{c} / \Upsilon_{e}=1.1,1.0$ and 0.8 , respectively. 


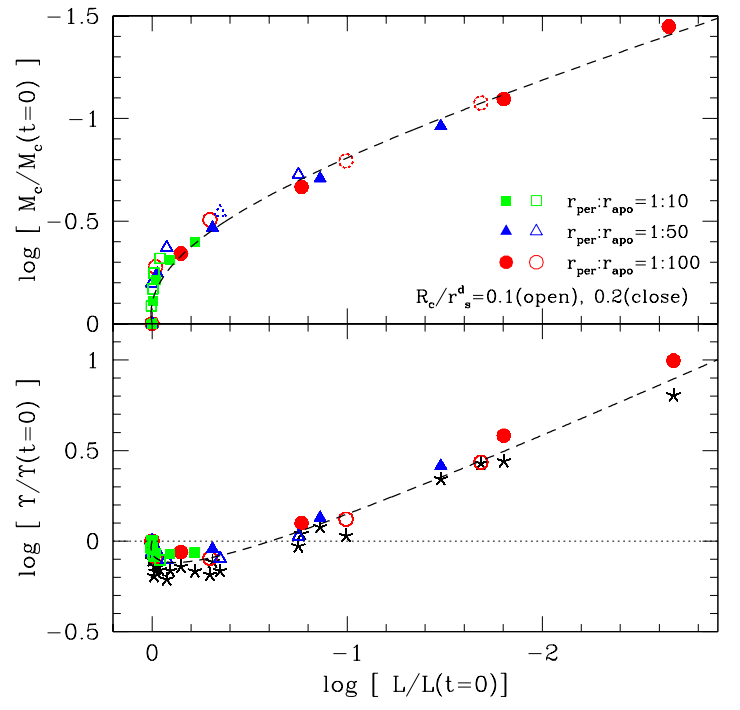

FIG. 7.- Evolution of the total bound mass within the (initial) luminous radius (upper panel) and of the mass-to-light ratio (lower panel) as a function of stellar mass loss for different orbits and stellar segregations. Starred symbols denote the classical massto-light ratio estimate $\Upsilon_{c} \propto \sigma_{0}^{2} /\left(\Sigma_{0} R_{h}\right)$ of Richstone \& Tremaine (1986). Other symbols are actual simulation measurements (within $R_{h}$ ). Note that the mass-to-light ratio increases when a dwarf undergoes substantial stripping of stars.

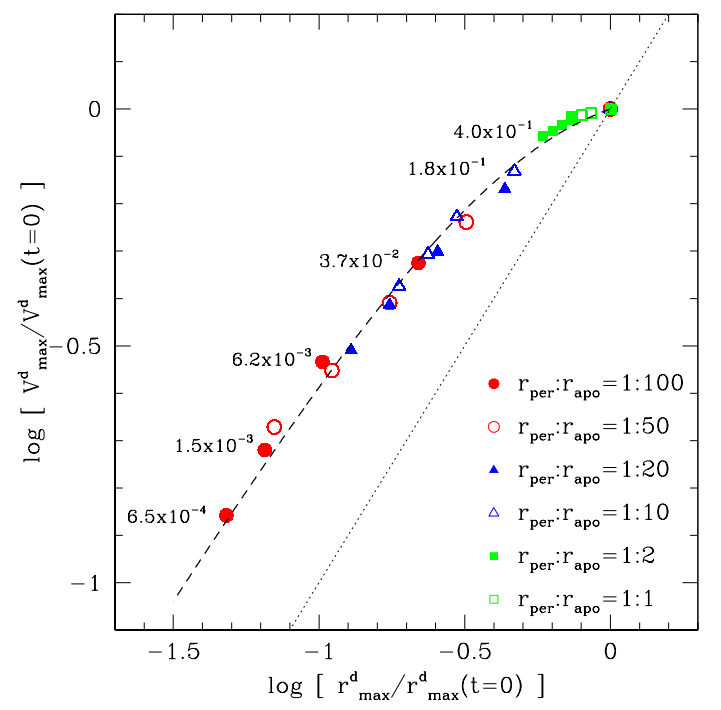

FIG. 8.- Evolution of the halo peak circular velocity $V_{\max } \equiv$ $V_{c}\left(r_{\max }\right)$ and its location, measured at the apocenter of the various orbits in our simulation series. The dotted line illustrates the roughly 1:1 relation expected between $V_{\max }$ and $r_{\max }$ for unperturbed $\Lambda \mathrm{CDM}$ halos. The dashed line is our fit to the evolutionary track using eq. 7 (see Table 21). Labels indicate approximately the total bound mass fraction along the curve.

Fig. 10 shows these parameters as a function of luminosity, using different symbols to highlight the various categories alluded to above. The symbols distinguish between Milky Way (blue squares) and M31 (red circles) dSphs, globular clusters (green dots), the dwarf ellipticals NGC 205, 147 and 185 (magenta stars), as well as the peculiar objects Willman I and Segue I (dot-centered squares). The isolated dwarph spheroidal Cetus is shown as a green crossed circle. (No comparable data are avail-

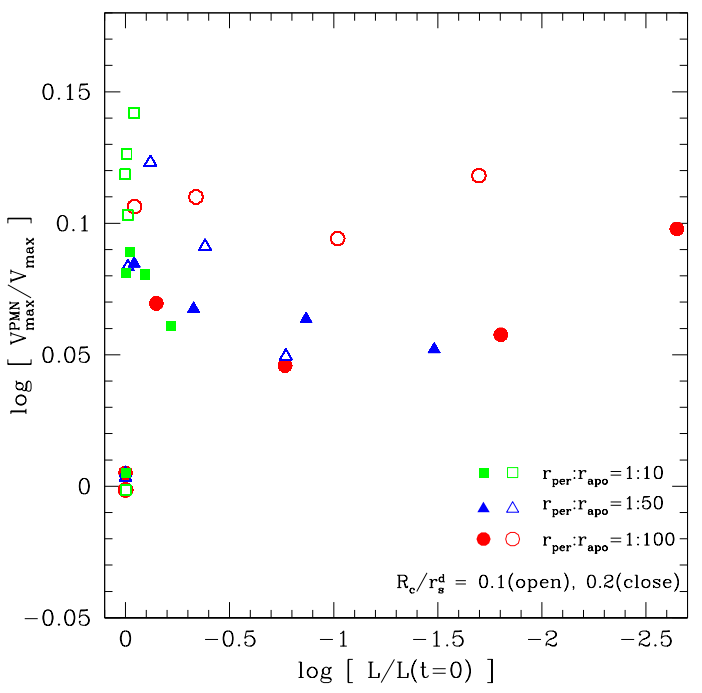

FIG. 9.- Estimates of $V_{\max }$ obtained adopting the procedure of Peñarrubia, McConnachie \& Navarro (2007, PMN), shown as a function of the bound stellar mass (or luminosity). This uses the King core radius and the central stellar velocity dispersion to estimate the mass within the luminous radius of the dwarf and then extrapolates this measurement assuming that the halo follows a $\Lambda$ CDM NFW profile. The estimates are given in units of the "true" $V_{\max }$ measured in our simulation series. Note that even in the case of extreme mass loss the PMN procedure yields robust estimates of the peak halo circular velocity.

able for Tucana.) Filled symbols correspond to the "classical" dSph population of the MW and M31; open symbols are used for the population of ultra-faint dwarfs.

\subsection{Tidal evolutionary tracks}

In each panel of Fig. 10 the dotted line indicates the predicted evolution of a dSph as it is stripped by tides. These "tidal tracks" have been computed using the empirical fits presented in Fig. 6. Each notch along the tracks corresponds to the consecutive loss of $90 \%$ of the stellar mass (or luminosity). The head of the track corresponds to the unperturbed object, whilst the arrowhead shows its (relative) position in each panel after losing $99.7 \%$ of its original stellar mass.

The tracks indicate the likely evolution of any dSph from its current location. Since it is unclear whether dSphs have not already been perturbed by tides, there is some uncertainty as to where the evolution of a given dSph would lie along its own "tidal track". An unperturbed dSph would evolve along a track constructed by translating the head of the track to its current location in each of the planes. In cases like Sagittarius, where some mass loss clearly has already taken place, the evolution is already underway along its "tidal track". Despite this uncertainty, a number of general inferences may be made in reference to the relative location of various systems in the space of parameters shown in Fig. 10

\subsection{Surface brightness evolution}

The first one concerns the evolution in surface brightness driven by tides. As shown by the tidal track in the top-left panel of Fig. 10, the surface brightness drops steeply as a dSph is stripped by tides: after losing the first half of its stars the surface brightness of a dSph 

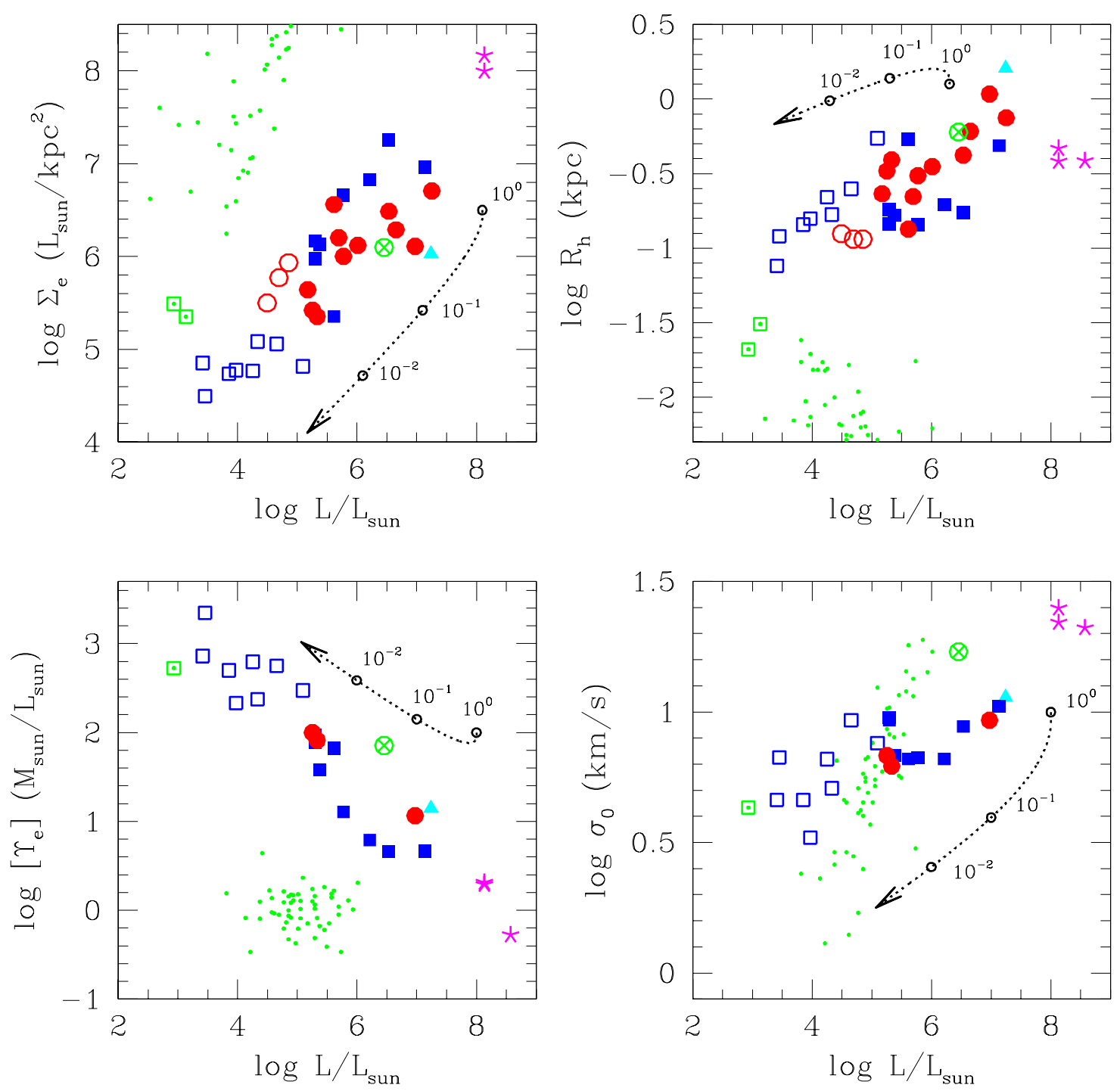

FIG. 10. - Local Group dSph structural parameters as a function of luminosity in the visual band. Data are compiled from the literature, as specified in Table 3 The panels show, respectively, the average surface brightness $\Sigma_{e} \equiv L /\left(2 \pi R_{h}^{2}\right)$; the half-light radius $R_{h}$; the mass-to-light ratio parameter, $\Upsilon_{e}=5 \sigma_{0}^{2} R_{h} / G L$; and the central projected velocity dispersion. Dwarf spheroidals of the Milky Way are shown with blue squares; those of M31 with red circles. We distinguish between the bright, "classical" population of dSphs (solid symbols, brighter than Draco) and "ultra-faint" dSphs (open symbols, fainter than Draco). This distinction is arbitrary and is made only to guide the discussion in Sec. 4 For illustration, we also show the Local Group dwarf ellipticals (magenta stars), the isolated Cetus dSph (green crossed circle) and the Milky Way globular clusters (green dots). Willman I and Segue I are shown with dotted green squares to highlight their intermediate properties between a dSph and a globular cluster. Finally, the Sagittarius dSph is shown with a full cyan triangle. Dotted lines correspond to the "tidal evolutionary tracks" that a dark matter-dominated dSph would follow as it is gradually stripped of stars. Each notch along the track corresponds to the conseccutive loss of $9 / 10$ of the stars.

drops by approximately a factor of $\sim 3$. This is intriguing, as it may explain why Sagittarius, the brightest of all dSphs in the Milky Way, is one of the faintest of the "classical" dSphs in terms of surface brightness. (Fornax, which has similar total luminosity as Sagittarius, is almost 10 times brighter in $\Sigma_{e}$.)

Interestingly, at the bright end, the classical dSph populations of M31 and MW also differ in their effective surface brightness. As noted by McConnachie \& Irwin (2006), the brightest $\left(L>10^{6} L_{\odot}\right)$ M31 dSphs are, at given luminosity, about a factor of 2 larger in $R_{h}$ and $\sim 4$ times fainter in surface brightness than their MW counterparts (compare, e.g., the brightest solid squares and solid circles in the top-left and top-right panels of Fig. 10). With the obvious caveat of small-number statistics, it is indeed puzzling that satellites formed around two seemingly normal spirals should exhibit such difference in structure. Could variations in the importance of tides be responsible for this offset?

The fact that tides tend to lower both the surface brightness and the half-light radius of dSphs simultaneously argues against this hypothesis. For example, if the lower surface brightness of M31 dwarfs (top-left panel) reflects the enhanced effect of tides on that population, then their progenitors must have had on average a larger half-light radius than at present, differentiating 


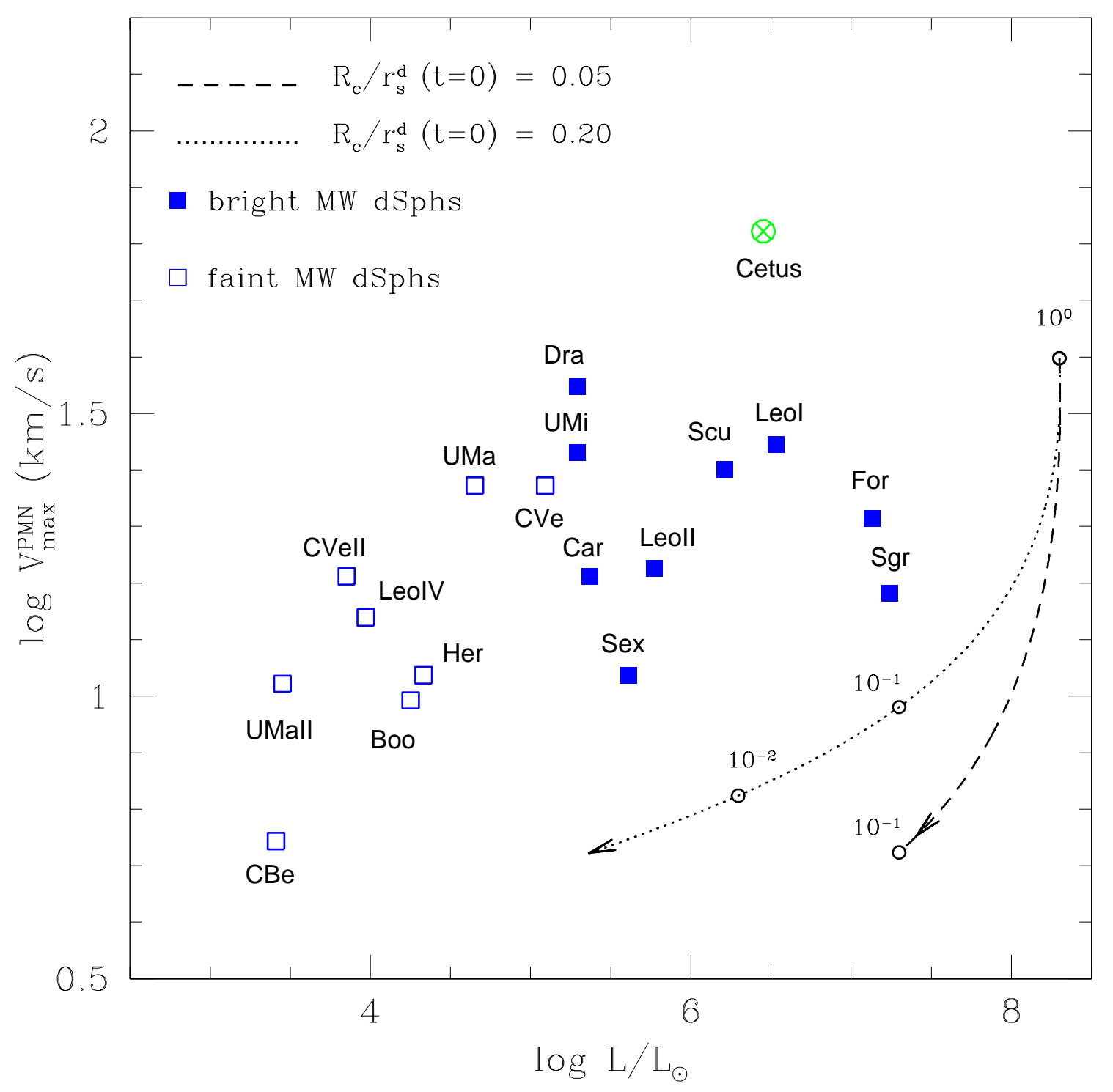

FIG. 11.- Peak halo circular velocity for all LG dSph (estimated following the procedure outlined by PMN) as a function of total luminosity. Symbols are as in Fig. 10 Curves show the expected evolution driven by tidal stripping, for two values of the stellar segregation, $R_{c} / r_{s}^{d}=0.05$ and 0.2 . The halos of ultra-faint dwarfs (open symbols) have on average peak circular velocities comparable to those of the "classical" dSphs (solid symbols). It is therefore unlikely that, as a whole, the faint dwarf population originates from stripping once-brighter progenitors like the "classical" dSphs. This does not exclude the possibility that some dSphs, and in particular those with low estimated $V_{\max }$, like CBe, may indeed be tidal remnants.

them even further from their MW counterparts.

Conversely, one could argue that the smaller size of the MW dSphs (top-right panel) might be the result from stronger tidal stripping. However, that would imply that their progenitors were on average brighter in surface brightness than the present-day M31 dSph population, again differentiating these systems even further. We conclude, therefore, that the differences in structure between the "classical" MW and M31 dSphs cannot be explained by differences in the importance of tides.

\subsection{Tidal tracks and scaling relations}

Another interesting result to note from Fig. 10 is that the "tidal track" in the $L-\Sigma_{e}$ plane is roughly parallel to the relation that holds for Local Group dSphs. Indeed, $\mathrm{a} \sim 10^{6} L_{\odot} \mathrm{dSph}$ that is stripped of $\sim 99 \%$ of its stars is expected to become a $\sim 10^{4} L_{\odot} \mathrm{dSph}$ two decades fainter in $\Sigma_{e}$. This is comparable to the observed trend, and suggests the intriguing possibility that Fornax-like or Draco-like systems, for example, might be the ancestors of some of the newly discovered ultra-faint MW dwarfs ${ }^{3}$ (shown as open blue squares in Fig. 10)

The plausibility of this suggestion is supported by the few available estimates of the dynamical mass-to-light ratio. This is shown by the "tidal track" in the $L-\Upsilon_{e}$

${ }^{3}$ Possible exceptions are Segue I and Willman I, which are considerably smaller than the rest of dSph of similar luminosity, a result that has led to speculation that these systems might actually be disrupted globular clusters rather than dwarf spheroidal galaxies. A recent measurement of the velocity dispersion of Willman 1 by Martin et al. (2007), however, indicates that Willman 1 has a very high mass-to-light ratio, implying a significant amount of dark matter and confirming its identity as a dwarf galaxy. 
panel of Fig. 10. The track again runs roughly parallel to the scaling observed for LG dSphs, suggesting that the extreme mass-to-light ratios of the newly-discovered dwarfs (at least those for which measurements are available) might in principle be due to tidal stripping of once brighter systems.

This would be a radically different interpretation of the origin of the main scaling relations of dSphs shown in Fig. 10, which is usually ascribed to the mass-dependent efficiency of star formation, feedback, and re-ionization in low-mass halos (see, e.g., Dekel \& Woo 2003).

One problem with the tidal interpretation, however, is that the $L-\sigma_{0}$ relation is much shallower than one would expect from the tidal evolutionary track, as shown in the bottom-right panel of Fig. 10. Indeed, there is little evidence for a correlation between luminosity and velocity dispersion for most dSphs, except perhaps for a hint of a downturn in the case of the faintest dwarfs.

A conservative conclusion, then, is that tides cannot induce the full range of observed parameters for dSphs, and that therefore the $\mathrm{LG} \mathrm{dSph}$ population must have been born with a significant range of properties. Extreme tidal stripping might be responsible for setting the properties of some individual dSphs, but are unlikely to explain the scaling relations of the whole $\mathrm{dSph}$ population.

\subsection{The halo properties of ultra-faint dSphs}

Support for this conclusion comes from comparing the properties of the dark halos of the ultra-faint dwarfs with those of brighter dSphs. If the ultra-faint dwarfs were originally massive systems that have lost a large fraction of their stellar mass, this would show as a net shift in the peak circular velocity, $V_{\max }$, between faint and bright dSphs. In quantitative terms, systems that have been stripped of $99 \%$ of their stars should have suffered an even higher loss of dark matter, and should have seen their $V_{\max }$ reduced by a factor of $\sim 3$ or so (see Fig. 8).

We explore this in the right-hand panel of Fig. 11 where we show the relation between dSph luminosity and inferred $V_{\max }$, computed adopting the procedure of PMN (see Sec. 3.7). This panel shows that, although on average ultra-faint dSphs have slightly lower values of $V_{\max }$, the difference is, again, not as large as one would expect if they were tidal remnants of the "classical" bright dSph population. This suggests again that it is unlikely that the population of faint dSphs descends directly from the bright dwarf spheroidals in the Milky Way, and that the observed correlations between the structural properties of dSphs are not the result of tidal stripping.

Fig. 11 also shows that the halos hosting dSphs are remarkably similar. Indeed, they differ by less than a factor of $\sim 3$ in $V_{\max }$ despite the fact that their luminous components span roughly 4 decades in luminosity. This result lends support to scenarios that envision dwarf galaxies as able to form only in halos above a certain mass threshold. Such a threshold has been suggested by the need to retain gas and to sustain continuing star formation despite the effects of feedback from evolving stars and the heating from photoionizing radiation (Efstathiou 1992, Bullock, Kravtsov \& Weinberg 2000, Somerville 2002, Benson et al. 2002).

In such a scenario, the scarcity of luminous dwarfs around the Milky Way may be reconciled with the copious substructure seen in N-body simulations of CDM halos (Moore et al 1999, Klypin et al 1999) by the fact that massive substructure halos that exceed the threshold are relatively scarce (Stoehr et al 2002, Hayashi et al 2003, Kazantzidis et al 2004). Indeed, only about $\sim 20$ substructure halos with $V_{\max }>20 \mathrm{~km} / \mathrm{s}$ are expected within the Milky Way halo (see PMN's Fig.8). This number is similar to the expected number of dSphs with such properties, once the number of newly-discovered dwarfs in the SDSS is corrected by the finite sky coverage of the survey (Koposov et al. 2007). Our results thus seem to support the main tenet of this interpretation: most dwarfs at the extreme faint end of the luminosity function inhabit relatively massive halos of mass similar to that defined by the threshold.

\section{SUMMARY}

We have used N-body simulations to study the dynamical evolution of dwarf spheroidal galaxies (dSphs) driven by galactic tides. We assume a cosmologically motivated scenario where dSphs are modelled as dark-matter dominated systems whose stellar component may be approximated as King models embedded within NFW halos. These systems are in eccentric orbits in the potential of a much more massive halo, also modelled using an NFW profile. Under these circumstances, tides operate nearly impulsively at pericenter and the dwarf relaxes quickly afterwards. We focus our analysis on the equilibrium structure reached by a dwarf following repeated episodes of mass loss. Our main conclusions may be summarized as follows.

- Our models show that NFW-embedded King models are extraordinarily resilient to tides; the density profile of the stellar component still resembles a King model even after losing more than $99 \%$ of its stars.

- The King-model structural parameters evolve as tides strip the galaxy. The stellar velocity dispersion, $\sigma_{0}$, central surface brightness, $\Sigma_{0}$, and core radius, $R_{c}$, decrease monotonically, in a manner controlled solely by the total amount of mass lost from within the luminous radius.

- The core radius, $R_{c}$, is the parameter least affected by tides: after losing $99 \%$ of the stars, $R_{c}$ decreases by only a factor of $\sim 2$. Thus, even in the event of extreme mass loss the core radius is a robust measure of the original size of the system.

- After substantial mass loss, tides tend to make dSphs more dark-matter dominated. This is because the tightly bound central dark matter "cusp" is more resilient to disruption than the comparatively more loosely bound "cored" King profile. Tidal effects may therefore help to explain the extremely large mass-to-light ratios of some of the newly-discovered ultra-faint Milky Way dwarfs.

- Although tides may induce changes in luminosity, surface brightness and mass-to-light ratio, that mimic the observed correlations, the weak (if any) luminosity dependence of the velocity dispersion implies that tides cannot be responsible for the scaling relations and full range of structural parameters of the Local Group dSphs. 
- The above conclusion is supported by the large peak circular velocities $\left(V_{\max }\right)$ inferred for the ultra-faint dSphs. With few exceptions, these are comparable to those of the bright dSphs, implying that it is unlikely that they are the true descendents of the present-day population of bright dSphs.

- Despite the large range in luminosity spanned by dSphs, they seem to inhabit halos with a surprisingly narrow range of peak circular velocity (or mass). This provides support for a scenario where dwarf galaxies are only able to form in halos above a certain mass threshold.

This is clearly a fast moving field where the tentative conclusions listed above are likely to be superseded as new data becomes available. In particular, spatially- resolved dynamical data for the newly-discovered dwarfs, as well as kinematic data for M31 dSphs should prove invaluable in order to constrain further current theoretical models of the formation of dSphs; the faintest galaxies in the Local Universe and certainly a puzzling collection of extragalactic elves.

This work has been supported by various grants to JFN from Canada's NSERC. JFN acknowledges useful discussions with Simon White, as well as the hospitality of the Max-Planck Institute for Astrophysics in Garching bei Muenchen, Germany. Stelios Kazantzidis is kindly thanked for providing the code used to generate the spherical NFW equilibrium N-body models.

\section{REFERENCES}

Aguilar, L. A., \& White, S. D. M. 1985, ApJ, 295, 374

Aguilar, L. A., \& White, S. D. M. 1986, ApJ, 307, 97

Battaglia, G., Tolstoy, E., Helmi, A., Irwin, M. J., Letarte, B., Jablonka, P., Hill, V., Venn, K. A., Shetrone, M. D., Arimoto, N., Primas, F., Kaufer, A., Francois, P.. Szeifert, T., Abel, T., \& Sadakane, K. 2006, astro-ph/0608370

Belokurov, V., Evans, N. W., Irwin, M. J., Hewett, P. C., \&

Wilkinson, M. I. 2006a, ApJ, 637, L29

Belokurov, V., et al. 2006b, ApJ, 647, L111

Belokurov, V., et al. 2007, ApJ, 654, 897

Benson, A. J., Lacey, C. G., Baugh, C. M., Cole, S., \& Frenk, C. S. 2002, MNRAS, 333, 156

Bryan G., Norman M., 1998, ApJ, 495, 80

Bullock J., Kravtsov A., Weinberg D., 2000, ApJ, 539, 517

Bullock, J. S., Kolatt, T. S., Sigad, Y., Somerville, R. S., Kravtsov, A. V., Klypin, A. A., Primack, J. R., \& Dekel, A. 2001, MNRAS, 321, 559

Bullock J.S., Johnston K.V., 2005, ApJ, 635, 931

Chapman, S. C., Ibata, R., Lewis, G. F., Ferguson, A. M. N., Irwin, M., McConnachie, A., \& Tanvir, N. 2005, ApJ, 632, L87

Chapman, S. C., et al. 2007, ApJ, 662, L79

Cole, S., Aragon-Salamanca, A., Frenk, C. S., Navarro, J. F., \& Zepf, S. E. 1994, MNRAS, 271, 781

Coleman, M. G., et al. 2007, ArXiv e-prints, 706, arXiv:0706.1669

Dekel, A., \& Woo, J. 2003, MNRAS, 344, 1131

Efstathiou, G. 1992, MNRAS, 256, 43P

Eke, V. R., Cole, S., \& Frenk, C. S. 1996, MNRAS, 282, 263

Eke, V. R., Navarro, J. F., \& Steinmetz, M. 2001, ApJ, 554, 114

Fellhauer M., Kroupa P., Baumgardt H., Bien R., Boily C. M., Spurzem R., Wassmer N., 2000, NewA, 5, 305

Fellhauer, M.,'\& Kroupa, P. 2006, MNRAS, 367, 1577

Gilmore, G., Wilkinson, M. I., Wyse, R. F. G., Kleyna, J. T., Koch, A., Evans, N. W., \& Grebel, E. K. 2007, ApJ, 663, 948

Harbeck, D., Gallagher, J. S., Grebel, E. K., Koch, A., \& Zucker, D. B. 2005, ApJ, 623, 159

Hayashi, E., Navarro, J. F., Taylor, J. E., Stadel, J., \& Quinn, T. 2003, ApJ, 584, 541

Ibata, R. A., Gilmore, G., \& Irwin, M. J. 1994, Nature, 370, 194

Ibata, R. A.. Gilmore, G., \& Irwin, M. J. 1995, MNRAS, 277, 781

Ibata, R., Chapman, S., Irwin, M., Lewis, G., \& Martin, N. 2006, MNRAS, 373, L70

Ibata, R., Martin, N. F., Irwin, M., Chapman, S., Ferguson, A. M. N., Lewis, G. F., \& McConnachie, A. W. 2007, ArXiv e-prints, 704, arXiv:0704.1318

Irwin M., Hatzidimitriou D., 1995, MNRAS, 277, 1354

Johnston, K. V., Choi, P. I., \& Guhathakurta, P. 2002, AJ, 124 127

Kazantzidis S., Magorrian J., Moore B., 2004, ApJ, 601, 37

Kazantzidis S., Zenter A., Kravtsov A., 2006, ApJ, 641, 647

Kauffmann, G., White, S. D. M., \& Guiderdoni, B. 1993, MNRAS, 264, 201

King, I. 1962, AJ, 67, 471

King, I. R. 1966, AJ, 71, 64

Kleyna, J. T., Wilkinson, M. I., Evans, N. W., \& Gilmore, G. 2005, ApJ, 630, L141

Klimentowski, J., Łokas, E. L., Kazantzidis, S., Prada, F., Mayer, L., \& Mamon, G. A. 2007, MNRAS, 378, 353

Klypin, A., Kravtsov, A. V., Valenzuela, O., \& Prada, F. 1999, ApJ, 522,82
Koch, A., Wilkinson, M. I., Kleyna, J. T., Gilmore, G. F., Grebel, E. K., Mackey, A. D., Evans, N. W., \& Wyse, R. F. G. 2007a, ApJ, 657, 241

Koch, A., Kleyna, J. T., Wilkinson, M. I., Grebel, E. K., Gilmore, G. F., Evans, N. W., Wyse, R. F. G., \& Harbeck, D. R. 2007b, AJ, 134,566

Koposov, S., et al. 2007, ArXiv e-prints, 706, arXiv:0706.2687

Kroupa, P. 1997, New Astronomy, 2, 139

Lahav, O., Lilje, P. B., Primack, J. R., \& Rees, M. J. 1991, MNRAS, 251, 128

Lewis, G. F., Ibata, R. A., Chapman, S. C., McConnachie, A., Irwin, M. J., Tolstoy, E., \& Tanvir, N. R. 2007, MNRAS, 375, 1364

Majewski, S. R., Skrutskie, M. F., Weinberg, M. D., \& Ostheimer, J. C. 2003, ApJ, 599, 1082

Majewski. S. R.. et al. 2007, ArXiv Astrophysics e-prints, arXiv:astro-ph/0702635

Martin, N. F., İbata, R. A., Irwin, M. J., Chapman, S., Lewis, G. F., Ferguson, A. M. N., Tanvir, N., \& McConnachie, A. W. 2006, MNRAS, 371, 1983

Martin, N. F., Ibata, R. A., Chapman, S. C., Irwin, M., \& Lewis, G. F. 2007a, ArXiv e-prints, 705, arXiv:0705.4622

Martínez-Delgado, D., Alonso-García, J., Aparicio, A., \& Gómez-Flechoso, M. A. 2001, ApJ, 549, L63

Mateo M.L., 1998, ARA\&A, 36, 435

Mateo, M., Olszewski, E. W.. \& Walker, M. G. 2007, ArXiv e-prints, 708, arXiv:0708.1327

Mayer, L., Kazantzidis, S., Mastropietro, C., \& Wadsley, J. 2007, Nature, 445,738

McConnachie, A. W., \& Irwin, M. J. 2006, MNRAS, 365, 1263

McConnachie, A. W., Arimoto, N., \& Irwin, M. 2007, MNRAS, 379,379

McConnachie, A. W., Peñarrubia, J., \& Navarro, J. F. 2007, MNRAS, L75

Moore, B., Ghigna, S., Governato, F., Lake, G., Quinn, T., Stadel, J., \& Tozzi, P. 1999, ApJ, 524, L19

Muñoz, R. R., et al. 2005, ApJ, 631, L137

Muñoz, R. R., et al. 2006, ApJ, 649, 201

Navarro, J. F. 1990, MNRAS, 242, 311

Navarro, J. F., Frenk, C. S., \& White, S. D. M. 1996, ApJ, 462, 563

Navarro J., Frenk C.S., White S.D.M., 1997, ApJ, 490, 493 (NFW)

Odenkirchen, M., et al. 2001, ApJ, 548, L165

Palma, C., Majewski, S. R., Siegel, M. H., Patterson, R. J., Ostheimer, J. C., \& Link, R. 2003, AJ, 125, 1352

Peñarrubia, J., Kroupa, P., \& Boily, C. M. 2002, MNRAS, 333, 779

Peñarrubia J., Just A., Kroupa P., 2004, MNRAS, 349, 747

Peñarrubia, J., \& Benson, A. J. 2005, MNRAS, 364, 977

Peñarrubia, J., Benson, A. J., Martínez-Delgado, D., \& Rix,

H. W. 2006, ApJ, 645, 240

Peñarrubia, J., McConnachie. A.. \& Navarro. J. F. 2007, ArXiv Astrophysics e-prints, arXiv:astro-ph/0701780 (PMN)

Power, C., Navarro, J. F., Jenkins, A., Frenk, C. S., White, S. D. M., Springel, V., Stadel, J., \& Quinn, T. 2003, MNRAS, 338,14

Read, J. I., Wilkinson, M. I., Evans, N. W., Gilmore, G., \&

Kleyna, J. T. 2006, MNRAS, 367, 387

Richstone D., Tremaine S., 1986, AJ, 92, 72 
Sales, L. V., Navarro, J. F., Abadi, M. G., \& Steinmetz, M. 2007, MNRAS, 379, 1475

Simon, J. D., \& Geha, M. 2007, ArXiv e-prints, 706 arXiv:0706.0516

Somerville, R. S. 2002, ApJ, 572, L23

Spergel. D. N.. et al. 2006, ArXiv Astrophysics e-prints,

arXiv:astro-ph/0603449

Strigarı, L. E., Bullock, J. S., \& Kaplinghat, M. 2007, ApJ, 657, L1

Tolstoy, E. et al. 2004, ApJ, 617, L119

Walker, M. G., Mateo, M., Olszewski, E. W., Pal, J. K., Sen, B., \& Woodroofe, M. 2006, ApJ, 642, L41
Walker, M. G., Mateo, M., Olszewski, E. W., Bernstein, R., Sen, B., \& Woodroofe, M. 2007, ApJS, 171, 389

Walsh, S. M., Jerjen, H., \& Willman, B. 2007, ApJ, 662, L83

Wilkinson, M. I., Kleyna, J., Evans, N. W., \& Gilmore, G. Irwin M.J., Grebel E.K., 2004, ApJ, 611, L21

Willman, B., et al. 2005a, AJ, 129, 2692

Willman, B., et al. 2005b, ApJ, 626, L85

Zucker, D. B., et al. 2006a, ApJ, 643, L103

Zucker, D. B., et al. 2006b, ApJ, 650, L41

Zucker, D. B., et al. 2007, ApJ, 659, L21 
TABLE 1

ORBITAL PARAMETERS OF OUR MODELS

\begin{tabular}{lcccc}
\hline \hline$r_{\text {per }}: r_{\text {apo }}$ & $\begin{array}{c}r_{\text {per }} \\
{[\mathrm{kpc}]}\end{array}$ & $\begin{array}{c}T_{r} \\
{[\mathrm{Gyr}]}\end{array}$ & $\begin{array}{c}r_{\text {tid }} \\
{\left[r_{s}^{d}\right]}\end{array}$ & $\begin{array}{c}M^{d}\left(<r_{\text {tid }}\right) \\
{\left[M_{\text {tot }}^{d}\right]}\end{array}$ \\
\hline $1: 1$ & 180.0 & $\infty$ & 9.75 & $6.4 \times 10^{-1}$ \\
$1: 2$ & 90.0 & 4.77 & 4.65 & $3.9 \times 10^{-1}$ \\
$1: 10$ & 18.0 & 3.36 & 0.70 & $5.2 \times 10^{-2}$ \\
$1: 20$ & 9.0 & 3.08 & 0.28 & $1.2 \times 10^{-2}$ \\
$1: 50$ & 3.6 & 2.94 & 0.08 & $1.3 \times 10^{-3}$ \\
$1: 100$ & 1.8 & 2.80 & 0.03 & $2.7 \times 10^{-4}$ \\
\hline
\end{tabular}

TABLE 2

EMPIRICAL FIT PARAMETERS TO THE TIDAL EVOLUTIONARY TRACKS (EQ. 7)

\begin{tabular}{cccccccc}
\hline \hline & $V_{\max }$ & $\Sigma_{0}$ & $R_{c}$ & $c_{K}$ & $\sigma_{0}$ & $L$ & $\Upsilon$ \\
& $\left(r_{\max }\right)$ & $\left(M_{c}\right)$ & $\left(M_{c}\right)$ & $\left(M_{c}\right)$ & $\left(M_{c}\right)$ & $\left(M_{c}\right)$ & $\left(M_{c}\right)$ \\
\hline$\alpha$ & 1.55 & 2.70 & 1.50 & 1.75 & 0.00 & 7.00 & -4.80 \\
$\beta$ & 0.99 & 2.00 & 0.65 & 0.00 & 0.50 & 3.30 & -1.60 \\
\hline
\end{tabular}


TABLE 3

Structural Parameters of Local Group Satellites

\begin{tabular}{|c|c|c|c|c|c|c|c|}
\hline Galaxy & $\begin{array}{c}\log _{10} L \\
\left.L_{\odot}\right]\end{array}$ & $\begin{array}{c}R_{h} \\
{[\mathrm{kpc}]}\end{array}$ & $\begin{array}{c}\log _{10} \Sigma_{e} \\
{\left[M_{\odot} \mathrm{kpc}^{-2}\right]}\end{array}$ & $\begin{array}{c}\sigma_{0} \\
{[\mathrm{~km} / \mathrm{s}]}\end{array}$ & $\begin{array}{c}\Upsilon_{e} \\
M_{\odot} / L_{\odot}\end{array}$ & $\begin{array}{l}\text { Symbol } \\
\text { [Fig. 10 }\end{array}$ & Refs. \\
\hline Sagittarius & 7.24 & 1.61 & 6.03 & 11.4 & 14.0 & solid triangle & (1) \\
\hline Fornax & 7.13 & 0.49 & 6.96 & 10.5 & 4.6 & solid square & $(2),(3)$ \\
\hline Leo I & 6.53 & 0.17 & 7.26 & 8.8 & 4.6 & " & $(2),(3)$ \\
\hline Sculptor & 6.21 & 0.20 & 6.83 & 6.6 & 6.14 & " & $(2),(3)$ \\
\hline Leo II & 5.77 & 0.14 & 6.66 & 6.7 & 12.7 & " & $(2),(3)$ \\
\hline Sextans & 5.61 & 0.54 & 5.35 & 6.6 & 66.7 & " & $(2),(3)$ \\
\hline Carina & 5.37 & 0.17 & 6.13 & 6.8 & 38.0 & " & $(2),(3)$ \\
\hline Ursa Minor & 5.29 & 0.18 & 5.98 & 9.3 & 93.1 & " & $(2),(3)$ \\
\hline Draco & 5.29 & 0.15 & 6.17 & 9.5 & 77.9 & " & $(2),(3)$ \\
\hline Canes Venatici & 5.09 & 0.55 & 4.82 & 7.6 & 297.9 & open square & $(6,12,15)$ \\
\hline Ursa Major & 4.65 & 0.25 & 5.06 & 9.3 & 561.6 & " & $(5,13,14,15)$ \\
\hline Hercules & 4.33 & 0.17 & 5.08 & 5.1 & 237.1 & " & $(9,15)$ \\
\hline Bootes & 4.25 & 0.22 & 4.77 & 6.6 & 625.2 & " & $(8,11,14)$ \\
\hline Leo IV & 3.97 & 0.16 & 4.77 & 3.3 & 213.9 & " & $(9,15)$ \\
\hline Canes Venatici II & 3.85 & 0.14 & 4.74 & 4.6 & 499.3 & " & $(9,15)$ \\
\hline Ursa Major II & 3.45 & 0.12 & 4.49 & 6.7 & 2217.0 & " & $(7,14,15)$ \\
\hline Coma Berenices & 3.41 & 0.08 & 4.85 & 4.6 & 725.8 & " & $(9,15)$ \\
\hline And VII & 7.25 & 0.75 & 6.71 & - & - & solid circle & (16) \\
\hline And II & 6.97 & 1.10 & 6.11 & 9.3 & 11.6 & " & $(16)$ \\
\hline And I & 6.65 & 0.61 & 6.29 & - & - & " & (16) \\
\hline And VI & 6.53 & 0.42 & 6.49 & - & - & " & (16) \\
\hline And III & 6.01 & 0.35 & 6.12 & - & - & " & (16) \\
\hline And V & 5.77 & 0.31 & 6.00 & - & - & " & (16) \\
\hline And XV & 5.69 & 0.22 & 6.20 & - & 一 & " & (20) \\
\hline And XVI & 5.61 & 0.13 & 6.56 & - & - & " & (20) \\
\hline And XIV & 5.33 & 0.39 & 5.35 & 6.2 & 81.3 & " & $(21)$ \\
\hline And IX & 5.25 & 0.33 & 5.42 & 6.8 & 99.2 & " & $(17,22)$ \\
\hline And $\mathrm{X}$ & 5.17 & 0.23 & 5.64 & - & - & “ & $(18)$ \\
\hline And XI & 4.85 & 0.11 & 5.93 & - & - & open circle & (19) \\
\hline And XIII & 4.69 & 0.12 & 5.77 & - & - & " & (19) \\
\hline And XII & 4.49 & 0.13 & 5.50 & - & - & $"$ & (19) \\
\hline Segue I & 3.13 & 0.03 & 5.35 & - & - & dotted square & (9) \\
\hline Willman I & 2.93 & 0.02 & 5.49 & 4.3 & 529.2 & " & $(4,14)$ \\
\hline Cetus & 6.45 & 0.60 & 6.01 & 17.0 & 71.4 & crossed circle & $(16,24)$ \\
\hline
\end{tabular}

References. - (1) Majewski et al. (2003); (2) Mateo (1998); (3) Irwin \& Hatzidimitriou (1995); (4) Willman (2005a); (5) Willman et al. (2005b); (6) Zucker et al. (2006a); (7) Zucker et al. (2006b); (8) Belokurov et al. (2006b); (9) Belokurov et al. (2007); (10) Walsh et al. (2007); (11) Muñoz et al. (2006); (12) Ibata et al. (2006); (13) Kleyna et al. (2005); (14) Martin et al. (2007); (15) Simon \& Geha (2007); (16) McConnachie\& Irwin (2006); (17) Harbeck et al. (2005); (18) Zucker et al. (2007); (19) Martin et al. (2006); (20) Ibata et al. (2007); (21) Majewski et al. (2007); (22) Chapman et al. (2005); (23) Coleman et al. (2007); (24) Lewis et al. (2007) 